\title{
Weak Convergence of Topological Measures
}

\author{
Svetlana V. Butler ${ }^{1}$ (1)
}

Received: 3 April 2020 / Revised: 26 March 2021 / Accepted: 30 March 2021 / Published online: 24 April 2021 (c) The Author(s) 2021

\begin{abstract}
Topological measures and deficient topological measures are defined on open and closed subsets of a topological space, generalize regular Borel measures, and correspond to (nonlinear in general) functionals that are linear on singly generated subalgebras or singly generated cones of functions. They lack subadditivity, and many standard techniques of measure theory and functional analysis do not apply to them. Nevertheless, we show that many classical results of probability theory hold for topological and deficient topological measures. In particular, we prove a version of Aleksandrov's theorem for equivalent definitions of weak convergence of deficient topological measures. We also prove a version of Prokhorov's theorem which relates the existence of a weakly convergent subsequence in any sequence in a family of topological measures to the characteristics of being a uniformly bounded in variation and uniformly tight family. We define Prokhorov and Kantorovich-Rubenstein metrics and show that convergence in either of them implies weak convergence of (deficient) topological measures on metric spaces. We also generalize many known results about various dense and nowhere dense subsets of deficient topological measures. The present paper constitutes a necessary step to further research in probability theory and its applications in the context of (deficient) topological measures and corresponding nonlinear functionals.
\end{abstract}

Keywords Weak convergence · Topological measure - Aleksandrov's theorem · Prokhorov's theorem · Prokhorov and Kantorovich-Rubenstein metrics · Dense subset

Mathematics Subject Classification (2020) 60B10 $\cdot 60 \mathrm{~B} 05 \cdot 28 \mathrm{~A} 33 \cdot 28 \mathrm{C} 15$

Svetlana V. Butler

svetbutler@gmail.com; svbutler@ucsb.edu

1 Department of Mathematics, University of California, Santa Barbara, 552 University Rd, Isla Vista, CA 93117, USA 


\section{Introduction}

The origins of the theory of quasi-linear functionals and topological measures lie in mathematical axiomatization and interpretations of quantum physics [28,32,33,42]. In J. von Neumann's axiomatization of quantum mechanics, physical observables can be represented by the space $\mathcal{L}$ of Hermitian operators on a complex Hilbert space. The state of a physical system is represented by a positive normalized linear functional on $\mathcal{L}$. Some physicists, however, argued that the linearity of the functional, $\rho(A+B)=$ $\rho(A)+\rho(B), A, B \in \mathcal{L}$, makes sense if observables $A$ and $B$ are simultaneously measurable, which means that $A, B$ are polynomials of the same $C \in \mathcal{L}$, so $A, B$ belong to the subalgebra of $\mathcal{L}$ generated by $C$. Mathematical interpretations of quantum physics by G. W. Mackey and R. V. Kadison led to very interesting mathematical problems, including the extension problem for probability measures in von Neumann algebras. This extension problem may be regarded as a special case of the linearity problem for physical states, which is closely related to the existence of quasi-linear functionals. J. F. Aarnes [3] introduced quasi-linear functionals (that are not linear) on $C(X)$ for a compact Hausdorff space $X$ and corresponding set functions, generalizing measures (initially called quasi-measures, now topological measures). He connected the two by establishing a representation theorem. Aarnes's quasi-linear functionals are functionals that are linear on singly generated subalgebras, but (in general) not linear. For more information about physical interpretation of quasi-linear functionals, see $[1-3,23-25,36]$.

M. Entov and L. Polterovich first linked the theory of quasi-linear functionals to symplectic geometry. They introduced symplectic quasi-states and partial symplectic quasi-states [23], which are subclasses of quasi-linear functionals. (On a symplectic manifold that is a closed oriented surface, every normalized quasi-linear functional is a symplectic quasi-state, see [36, Chapter 5]). Article [23] was followed by numerous papers and a monograph [36], and many authors have investigated and used various aspects of symplectic quasi-states and topological measures: their properties, their connection to spectral numbers and homogeneous quasi-morphisms, ways of constructing and approximating symplectic quasi-states, etc. Symplectic quasi-states can be used as a measurement of Poisson commutativity, and topological measures can be used to distinguish Lagrangian knots that have identical classical invariants [23, Chapters 4,6]. Symplectic quasi-states and topological measures play an important role in function theory on symplectic manifolds.

Deficient topological measures are generalizations of topological measures. They were first defined and used by A. Rustad and O. Johansen [27] and later independently reintroduced and further developed by M. Svistula [39,40]. Deficient topological measures are not only interesting by themselves, but also provide an essential framework for studying topological measures and quasi-linear functionals. Topological measures and deficient topological measures generalize regular Borel measures and correspond to functionals that are linear on singly generated subalgebras or singly generated cones of functions. These nonlinear functionals can be described in several ways, including symmetric and asymmetric Choquet integrals, see [20, pp. 62, 87] and [15, Corollary 8.5, Theorem 8.7, Remark 8.11]. Deficient topological measures are not supermodular, and their domains are not closed under intersection and union; for these and 
other reasons, results of Choquet theory do not automatically translate for functionals representing deficient topological measures. It is interesting that, with different proof methods, one may obtain results that are typical for, stronger than, or strikingly different from Choquet theory results.

Topological measures and deficient topological measures are defined on open and closed subsets of a topological space, which means that there is no algebraic structure on the domain. They lack subadditivity and other properties typical for measures, and many standard techniques of measure theory and functional analysis do not apply to them. Nevertheless, we show that many classical results of probability theory hold for topological and deficient topological measures. In particular, we prove versions of Aleksandrov's theorem for equivalent definitions of weak convergence of topological and deficient topological measures. We also prove a version of Prokhorov's theorem which relates the existence of a weakly convergent subsequence in any sequence in a family of topological measures to the characteristics of being a uniformly bounded in variation and uniformly tight family. We define Prokhorov and Kantorovich-Rubenstein metrics and show that convergence in either of them implies weak convergence of deficient topological measures. We also generalize many known results about various dense and nowhere dense subsets of deficient topological measures.

The present paper constitutes a necessary step to further research in probability theory and its applications in the context of topological measures and corresponding nonlinear functionals.

In this paper, $X$ is a locally compact Hausdorff space. By $C(X)$, we denote the set of all real-valued continuous functions on $X$ with the uniform norm, by $C_{0}(X)$ the set of continuous functions on $X$ vanishing at infinity, by $C_{c}(X)$ the set of continuous functions with compact support, and by $C_{0}^{+}(X), C_{c}^{+}(X), C^{+}(X)$ the collections of all nonnegative functions from $C_{0}(X), C_{c}(X), C(X)$, respectively.

When we consider maps into extended real numbers, we assume that any such map is not identically $\infty$.

We denote by $\bar{E}$ the closure of a set $E$, and by $\sqcup$ a union of disjoint sets. A set $A \subseteq X$ is called bounded if $\bar{A}$ is compact. We denote by $i d$ the identity function $i d(x)=x$, and by $1_{K}$ the characteristic function of a set $K$. By supp $f$ we mean $\{x: f(x) \neq 0\}$. We say that $Y$ is dense in $Z$ if $Z \subseteq \bar{Y}$.

Several collections of sets are used often. They include: $\mathscr{O}(X) ; \mathscr{C}(X)$; and $\mathscr{K}(X)-$ the collection of open subsets of $X$; the collection of closed subsets of $X$; and the collection of compact subsets of $X$, respectively.

Definition 1.1 Let $X$ be a topological space and $v$ be a set function on a family $\mathcal{E}$ of subsets of $X$ that contains $\mathscr{O}(X) \cup \mathscr{C}(X)$ with values in $[0, \infty]$. We say that

$-v$ is compact-finite if $v(K)<\infty$ for any $K \in \mathscr{K}(X)$;

$-v$ is simple if it only assumes values 0 and 1 ;

$-v$ is finite if $v(X)<\infty$;

$-v$ is inner regular (or inner compact regular) if $v(A)=\sup \{v(C): C \subseteq A, C \in$ $\mathscr{K}(X)\}$ for $A \in \mathcal{E}$;

- $v$ is inner closed regular if $v(A)=\sup \{v(C): C \subseteq A, C \in \mathscr{C}(X)\}$ for $A \in \mathcal{E}$;

$-v$ is outer regular if $v(A)=\inf \{v(U): A \subseteq U, U \in \mathscr{O}(X)\}$ for $A \in \mathcal{E}$. 
Definition 1.2 A measure on $X$ is a countably additive set function on a $\sigma$-algebra of subsets of $X$ with values in $[0, \infty]$. A Borel measure on $X$ is a measure on the Borel $\sigma$-algebra on $X$. A Radon measure $m$ on $X$ is a compact-finite Borel measure that is outer regular on all Borel sets, and inner regular on all open sets, i.e., $m(K)<\infty$ for every compact $K, m(E)=\inf \{m(U): E \subseteq U, U$ is open $\}$ for every Borel set $E$, and $m(U)=\sup \{m(K): K \subseteq U, K$ is compact $\}$ for every open set $U$. For a Borel measure $m$ that is inner regular on all open sets (in particular, for a Radon measure), we define supp $m$, the support of $m$, to be the complement of the largest open set $W$ such that $m(W)=0$.

For the following fact, see, for example, [22, Chapter XI, 6.2] and [19, Lemma 2.5].

Lemma 1.1 Let $K \subseteq U, K \in \mathscr{K}(X), U \in \mathscr{O}(X)$ in a locally compact space $X$. Then, there exists a set $V \in \mathscr{O}(X)$ such that $C=\bar{V}$ is compact and $K \subseteq V \subseteq \bar{V} \subseteq U$. If $X$ is also locally connected, and either $K$ or $U$ is connected, then $V$ and $C$ can be chosen to be connected.

Definition 1.3 A deficient topological measure on a locally compact space $X$ is a set function $v: \mathscr{C}(X) \cup \mathscr{O}(X) \longrightarrow[0, \infty]$ which is finitely additive on compact sets, inner compact regular, and outer regular, i.e.:

(DTM1) if $C \cap K=\emptyset, C, K \in \mathscr{K}(X)$, then $v(C \sqcup K)=v(C)+v(K)$;

(DTM2) $v(U)=\sup \{v(C): C \subseteq U, C \in \mathscr{K}(X)\}$ for $U \in \mathscr{O}(X)$;

(DTM3) $v(F)=\inf \{v(U): F \subseteq U, U \in \mathscr{O}(X)\}$ for $F \in \mathscr{C}(X)$.

Clearly, for a closed set $F, v(F)=\infty$ iff $v(U)=\infty$ for every open set $U$ containing $F$. If two deficient topological measures agree on compact sets (or on open sets), then they coincide.

Definition 1.4 A topological measure on $X$ is a set function $\mu: \mathscr{C}(X) \cup \mathscr{O}(X) \longrightarrow$ $[0, \infty]$ satisfying the following conditions:

(TM1) if $A, B, A \sqcup B \in \mathscr{K}(X) \cup \mathscr{O}(X)$, then $\mu(A \sqcup B)=\mu(A)+\mu(B)$;

(TM2) $\mu(U)=\sup \{\mu(K): K \in \mathscr{K}(X), K \subseteq U\}$ for $U \in \mathscr{O}(X)$;

(TM3) $\mu(F)=\inf \{\mu(U): U \in \mathscr{O}(X), F \subseteq U\}$ for $F \in \mathscr{C}(X)$.

By $\mathbf{D T M}(X)$ and $\mathbf{T M}(X)$, we denote, respectively, the collections of all finite deficient topological measures and all finite topological measures on $X$.

The following two theorems from [17, Sect. 4] give criteria for a deficient topological measure to be a topological measure or a measure.

Theorem 1.1 Let X be compact and $v$ a deficient topological measure. The following are equivalent:

(a) $v$ is a real-valued topological measure;

(b) $v(X)=v(C)+v(X \backslash C), \quad C \in \mathscr{C}(X)$;

(c) $v(X) \leq v(C)+v(X \backslash C), \quad C \in \mathscr{C}(X)$.

Let $X$ be locally compact and $v$ a deficient topological measure. The following are equivalent: 
(a) $v$ is a topological measure;

(b) $v(U)=v(C)+v(U \backslash C), \quad C \subseteq U, \quad C \in \mathscr{K}(X), \quad U \in \mathscr{O}(X)$;

(c) $v(U) \leq v(C)+v(U \backslash C), \quad C \subseteq U, \quad C \in \mathscr{K}(X), \quad U \in \mathscr{O}(X)$.

Theorem 1.2 Let $\mu$ be a deficient topological measure on a locally compact space $X$. The following are equivalent:

(a) If $C, K$ are compact subsets of $X$, then $\mu(C \cup K) \leq \mu(C)+\mu(K)$.

(b) If $U, V$ are open subsets of $X$, then $\mu(U \cup V) \leq \mu(U)+\mu(V)$.

(c) $\mu$ admits a unique extension to an inner regular on open sets, outer regular Borel measure $m$ on the Borel $\sigma$-algebra of subsets of X. $m$ is a Radon measure iff $\mu$ is compact-finite. If $\mu$ is finite, then $m$ is an outer regular and inner closed regular Borel measure.

Remark 1.1 Let $X$ be locally compact, and let $\mathscr{M}$ be the collection of all Borel measures on $X$ that are inner regular on open sets and outer regular on all Borel sets. Thus, $\mathscr{M}$ includes regular Borel measures and Radon measures. We denote by $M(X)$ the restrictions to $\mathscr{O}(X) \cup \mathscr{C}(X)$ of measures from $\mathscr{M}$, and by $\mathbf{M}(X)$ the set of all finite measures from $M(X)$. We have:

$$
M(X) \varsubsetneqq \operatorname{TM}(X) \varsubsetneqq \operatorname{DTM}(X)
$$

The inclusions follow from the definitions. When $X$ is compact, there are examples of topological measures that are not measures and of deficient topological measures that are not topological measures in numerous papers, beginning with [3,6,12,27], and [39]. When $X$ is locally compact, see [13], [17, Sects. 4, 5], and [19, Sect. 15] for more information on proper inclusion in (1), criteria for a deficient topological measure to be a measure from $M(X)$, and various examples. We also give some examples at the end of this section.

Remark 1.2 In [17, Sect. 3], we show that a deficient topological measure $v$ is $\tau$ smooth on compact sets (i.e., if a net $K_{\alpha} \searrow K$, where $K_{\alpha}, K \in \mathscr{K}(X)$, then $\left.\mu\left(K_{\alpha}\right) \rightarrow \mu(K)\right)$, and also $\tau$-smooth on open sets (i.e., if a net $U_{\alpha} \nearrow U$, where $U_{\alpha}, U \in \mathscr{O}(X)$, then $\left.\mu\left(U_{\alpha}\right) \rightarrow \mu(U)\right)$. In particular, a deficient topological measure is additive on open sets. A deficient topological measure $v$ is also superadditive, i.e., if $\bigsqcup_{t \in T} A_{t} \subseteq A$, where $A_{t}, A \in \mathscr{O}(X) \cup \mathscr{C}(X)$, and at most one of the closed sets (if there are any) is not compact, then $v(A) \geq \sum_{t \in T} v\left(A_{t}\right)$. If $F \in \mathscr{C}(X)$ and $C \in \mathscr{K}(X)$ are disjoint, then $v(F)+v(C)=v(F \sqcup C)$. One may consult [17] for more properties of deficient topological measures on locally compact spaces.

Definition 1.5 For a deficient topological measure $\mu$, we define $\|\mu\|=\mu(X)=$ $\sup \{\mu(K): K \in \mathscr{K}(X)\}$.

Definition 1.6 We call a functional $\rho$ on $C_{0}(X)$ with values in $[-\infty, \infty]$ (assuming at most one of $\infty,-\infty)$ and $|\rho(0)|<\infty$ a p-conic quasi-linear functional if

(p1) $f g=0, f, g \geq 0$ implies $\rho(f+g)=\rho(f)+\rho(g)$.

(p2) $0 \leq g \leq f$ implies $\rho(g) \leq \rho(f)$. 
(p3) For each $f$, if $g, h \in A^{+}(f), a, b \geq 0$, then $\rho(a g+b h)=a \rho(g)+b \rho(h)$. Here, $A^{+}(f)=\{\phi \circ f: \phi \in C(\overline{f(X)}), \phi$ is non-decreasing $\}$, (with $\phi(0)=0$ if $X$ is non-compact) is a cone generated by $f$.

For a functional $\rho$ on $C_{0}(X)$, we consider $\|\rho\|=\sup \{|\rho(f)|:\|f\| \leq 1\}$ and we say $\rho$ is bounded if $\|\rho\|<\infty$. Let $\boldsymbol{\Phi}^{+}\left(C_{0}^{+}(X)\right)$ be the set of all bounded p-conic quasi-linear $\rho$ functionals on $C_{0}^{+}(X)$.

A real-valued map $\rho$ on $C_{0}(X)$ is a quasi-linear functional (or a positive quasi-linear functional) if

(QI1) $f \geq 0 \Longrightarrow \rho(f) \geq 0$.

(QI2) $\rho(a f)=a \rho(f)$ for $a \in \mathbb{R}$.

(QI3) For each $f$, if $g, h \in B(f)$, then $\rho(h+g)=\rho(h)+\rho(g)$. Here, $B(f)=$ $\{\phi \circ f: \phi \in C(\overline{f(X)})\}$ (with $\phi(0)=0$ if $X$ is non-compact) is a subalgebra generated by $f$.

Remark 1.3 There is an order-preserving bijection between DTM $(X)$ and $\boldsymbol{\Phi}^{+}\left(C_{0}^{+}(X)\right)$. See [15, Sect. 8]. In particular, there is an order-preserving isomorphism between finite topological measures on $X$ and quasi-linear functionals on $C_{0}(X)$ of finite norm, and $\mu$ is a measure iff the corresponding functional is linear (see [15, Theorem 8.7], [37, Theorem 3.9], and [40, Theorem 15]). We outline the correspondence.

(I) Given a finite deficient topological measure $\mu$ on a locally compact space $X$ and $f \in C_{b}(X)$, define functions on $\mathbb{R}$ :

$$
\begin{aligned}
& R_{1}(t)=R_{1, \mu, f}(t)=\mu\left(f^{-1}((t, \infty))\right), \\
& R_{2}(t)=R_{2, \mu, f}(t)=\mu\left(f^{-1}([t, \infty))\right) .
\end{aligned}
$$

Let $r$ be the Lebesgue-Stieltjes measure associated with $-R_{1}$, a regular Borel measure on $\mathbb{R}$. The supp $r \subseteq \overline{f(X)}$. We define a functional on $C_{b}(X)$ (in particular, a functional on $\left.C_{0}(X)\right)$ :

$$
\begin{aligned}
\mathcal{R}(f) & =\int_{\mathbb{R}} \mathrm{id} \mathrm{d} r=\int_{[a, b]} \mathrm{id} \mathrm{d} r \\
& =\int_{a}^{b} R_{1}(t) \mathrm{d} t+a \mu(X)=\int_{a}^{b} R_{2}(t) \mathrm{d} t+a \mu(X) .
\end{aligned}
$$

where $[a, b]$ is any interval containing $f(X)$. If $f(X) \subseteq[0, b]$, we have:

$$
\mathcal{R}(f)=\int_{[0, b]} \mathrm{id} \mathrm{d} r=\int_{0}^{b} R_{1}(t) \mathrm{d} t=\int_{0}^{b} R_{2}(t) \mathrm{d} t .
$$

We call the functional $\mathcal{R}$ a quasi-integral (with respect to a deficient topological measure $\mu$ ) and write:

$$
\int_{X} f \mathrm{~d} \mu=\mathcal{R}(f)=\mathcal{R}_{\mu}(f)=\int_{\mathbb{R}} \mathrm{id} \mathrm{d} r .
$$


(II) Functional $\mathcal{R}$ is nonlinear. By [15, Lemma 7.7, Theorem 7.10, Lemma 3.6, Lemma 7.12], we have:

(a) $\mathcal{R}(f)$ is positive-homogeneous, i.e., $\mathcal{R}(c f)=c \mathcal{R}(f)$ for $c \geq 0, f \in C_{b}(X)$.

(b) $\mathcal{R}(0)=0$.

(c) $\mathcal{R}$ is monotone, i.e., if $f \leq g$, then $\mathcal{R}(f) \leq \mathcal{R}(g)$ for $f, g \in C_{b}(X)$.

(d) $\mu(X) \cdot \inf _{x \in X} f(x) \leq \mathcal{R}(f) \leq \mu(X) \cdot \sup _{x \in X} f(x)$ for $f \in C_{b}(X)$.

(e) If $f g=0$, where $f, g \geq 0$, then $\mathcal{R}(f+g)=\mathcal{R}(f)+\mathcal{R}(g)$ for $f, g \in C_{b}(X)$; if $f g=0$, where $f \geq 0, g \leq 0$ or $f, g \geq 0$, then $\mathcal{R}(f+g)=\mathcal{R}(f)+\mathcal{R}(g)$ for $f, g \in C_{0}(X)$.

(III) A functional $\rho$ with values in $[-\infty, \infty]$ (assuming at most one of $\infty,-\infty$ ) and $|\rho(0)|<\infty$ is called a d-functional if on nonnegative functions it is positivehomogeneous, monotone, and orthogonally additive, i.e., for $f, g \in D(\rho)$ (the domain of $\rho$ ), we have: (d1) $f \geq 0, a>0 \Longrightarrow \rho(a f)=a \rho(f)$; (d2) $0 \leq g \leq$ $f \Longrightarrow \rho(g) \leq \rho(f)$; (d3) $f \cdot g=0, f, g \geq 0 \Longrightarrow \rho(f+g)=\rho(f)+\rho(g)$. Let $\rho$ be a d-functional with $C_{c}^{+}(X) \subseteq D(\rho) \subseteq C_{b}(X)$. In particular, we may take functional $\mathcal{R}$ on $C_{0}^{+}(X)$. The corresponding deficient topological measure $\mu=\mu_{\rho}$ is given as follows:

If $U$ is open, $\mu_{\rho}(U)=\sup \left\{\rho(f): f \in C_{c}(X), 0 \leq f \leq 1\right.$, supp $\left.f \subseteq U\right\}$, if $F$ is closed, $\mu_{\rho}(F)=\inf \left\{\mu_{\rho}(U): F \subseteq U, U \in \mathscr{O}(X)\right\}$.

If $K$ is compact, $\mu_{\rho}(K)=\inf \left\{\rho(g): g \in C_{c}(X), g \geq 1_{K}\right\}=\inf \{\rho(g): g \in$ $\left.C_{c}(X), 1_{K} \leq g \leq 1\right\}$. (See [15, Sect. 5].)

If given a finite deficient topological measure $\mu$, we obtain $\mathcal{R}$, and then, $\mu_{\mathcal{R}}$, then $\mu=\mu_{\mathcal{R}}$.

Remark 1.4 Integrals with respect to (deficient) topological measures on a locally compact space $X$ have Lipschitz property: If $\mu$ is a finite deficient topological measure, $f, g \in C_{c}^{+}(X)$, supp $f$, supp $g \subseteq K, K$ is compact, then

$$
|\mathcal{R}(f)-\mathcal{R}(g)|=\left|\int_{X} f \mathrm{~d} \mu-\int_{X} g \mathrm{~d} \mu\right| \leq\|f-g\| \mu(K) .
$$

If $\mu$ is a finite topological measure, $f, g \in C_{0}(X)$, then

$$
\int_{X} f \mathrm{~d} \mu=\int_{X} f^{+} \mathrm{d} \mu-\int_{X} f^{-} \mathrm{d} \mu
$$

and

$$
\left|\int_{X} f \mathrm{~d} \mu-\int_{X} g \mathrm{~d} \mu\right| \leq 2\|f-g\| \mu(X) .
$$

See [15, Lemma 7.12, Theorem 8.7] and [18, Corollary 56].

We would like to give some examples. 
Definition 1.7 A set $A$ is bounded if $\bar{A}$ is compact. If $X$ is locally compact, noncompact, a set $A$ is solid if $A$ is connected, and $X \backslash A$ has only unbounded connected components. If $X$ is compact, a set $A$ is solid if $A$ and $X \backslash A$ are connected.

Many examples of topological measures that are not measures are obtained in the following way. Define a so-called solid-set function on bounded open solid and compact solid sets in a locally compact, connected, locally connected, Hausdorff space. A solid set function extends to a unique topological measure. See [5, Definition 2.3, Theorem 5.1], [19, Definition 6.1, Theorem 10.7].

Example 1.1 Suppose that $\lambda$ is the Lebesgue measure on $X=\mathbb{R}^{2}$, and the set $P$ consists of two points $p_{1}=(0,0)$ and $p_{2}=(2,0)$. For each bounded open solid or compact solid set $A$, let $v(A)=0$ if $A \cap P=\emptyset, v(A)=\lambda(A)$ if $A$ contains one point from $P$, and $v(A)=2 \lambda(X)$ if $A$ contains both points from $P$. Then, $v$ is a solid-set function (see [19, Example 15.5]), and $v$ extends to a unique topological measure on $X$. Let $K_{i}$ be the closed ball of radius 1 centered at $p_{i}$ for $i=1,2$. Then, $K_{1}, K_{2}$ and $C=K_{1} \cup K_{2}$ are compact solid sets, $v\left(K_{1}\right)=v\left(K_{2}\right)=\pi, v(C)=4 \pi$. Since $v$ is not subadditive, it cannot be a measure. The quasi-linear functional corresponding to $v$ is not linear.

Example 1.2 Let $X=\mathbb{R}^{2}$ or a square, $n$ be a natural number, and let $P$ be a set of distinct $2 n+1$ points. For each bounded open solid or compact solid set $A$, let $v(A)=i / n$ if $A$ contains $2 i$ or $2 i+1$ points from $P$. The set function $v$ defined in this way is a solid-set function, and it extends to a unique topological measure on $X$ that assumes values $0,1 / n, \ldots, 1$. See [4, Example 2.1], [6, Example 2.5], [12, Examples 4.14, 4.15], and [19, Example 15.9]. The resulting topological measure is not a measure. For instance, when $X$ is the square and $n=3$, it is easy to represent $X=A_{1} \cup A_{2} \cup A_{3}$, where each $A_{i}$ is a compact solid set containing one point from $P$. Then, $v\left(A_{i}\right)=0$ for $i=1,2,3$, while $v(X)=1$. Since $v$ is not subadditive, it is not a measure, and the quasi-linear functional $\rho$ corresponding to $v$ is not linear. In [18, Example 59], we take $n=5$ and show that there are $f, g \geq 0$ such that $\rho(f+g) \neq \rho(f)+\rho(g)$. If $X$ is locally compact, non-compact, for the functional $\rho$ we consider a new functional $\rho_{g}$ defined by $\rho_{g}(f)=\rho(g f)$, where $g \geq 0$. The new functional $\rho_{g}$ corresponds to a deficient topological measure obtained by integrating $g$ over closed and open sets with respect to a topological measure $v$. We can choose $g \geq 0$ or $g>0$ so that $\rho_{g}$ is no longer linear on singly generated subalgebras, but only linear on singly generated cones. See [16, Example 35, Theorem 43] for details.

Example 1.3 Let $X$ be locally compact, and let $D$ be a connected compact subset of $X$. Define a set function $v$ on $\mathscr{O}(X) \cup \mathscr{C}(X)$ by setting $v(A)=1$ if $D \subseteq A$ and $v(A)=0$ otherwise, for any $A \in \mathscr{O}(X) \cup \mathscr{C}(X)$. If $D$ has more than one element, then $v$ is a deficient topological measure, but not a topological measure. See [17, Example 6.1] and [40, Example 1, p.729] for details.

For more examples of topological measures and quasi-integrals on locally compact spaces, see $[13,18]$, and the last section of [19]. For more examples of deficient topological measures, see [17] and [40]. 


\section{Aleksandrov's Theorem for Deficient Topological Measures}

Definition 2.1 The weak topology on $\operatorname{DTM}(X)$ is the coarsest (weakest) topology for which maps $\mu \longmapsto \mathcal{R}_{\mu}(f), f \in C_{0}^{+}(X)$ are continuous.

The basic neighborhoods for the weak topology have the form

$$
\begin{gathered}
N\left(\nu, f_{1}, \ldots, f_{n}, \epsilon\right)=\left\{\mu \in \mathbf{D T M}(X):\left|\mathcal{R}_{\mu}\left(f_{i}\right)-\mathcal{R}_{v}\left(f_{i}\right)\right|<\epsilon, f_{i} \in C_{0}^{+}(X)\right. \\
i=1, \ldots, n\}
\end{gathered}
$$

Let $\mu_{\alpha}$ be a net in $\operatorname{DTM}(X), \mu \in \mathbf{D T M}(X)$. The net $\mu_{\alpha}$ converges weakly to $\mu$ (and we write $\mu_{\alpha} \Longrightarrow \mu$ ) iff $\mathcal{R}_{\mu_{\alpha}}(f) \rightarrow \mathcal{R}_{\mu}(f)$ for every $f \in C_{0}^{+}(X)$, i.e., $\int f d \mu_{\alpha} \rightarrow \int f d \mu$ for every $f \in C_{0}^{+}(X)$.

By [15, Theorem 8.7], DTM $(X)$ with weak convergence is homeomorphic to $\boldsymbol{\Phi}^{+}\left(C_{0}^{+}(X)\right)$ with pointwise convergence, and $\mathbf{T M}(X)$ is homeomorphic to the space of quasi-linear functionals with pointwise convergence.

Remark 2.1 Our definition of weak convergence corresponds to one used in probability theory. It is the same as a functional analytical definition of $w k *$ convergence on DTM $(X)$ (respectively, on TM(X)), which is justified by the fact that this topology agrees with the weak* topology induced by p-conic quasi-linear functionals (respectively, quasi-linear functionals). In many papers, the term " $w k^{*}$-topology" is used.

Definition 2.2 Let $\mu$ be a deficient topological measure. A set $A$ is called a $\mu$-continuity set if $\mu(\bar{A})=\mu\left(A^{o}\right)$.

Remark 2.2 In probability theory, with $\mu$ a measure, a set $A$ is called a $\mu$-continuity set if $\mu(\partial A)=0$. If $\mu$ is a measure (or $\mu$ is a topological measure and $\bar{A}$ is compact), this definition is equivalent to Definition 2.2. If $\mu$ is a deficient topological measure, then by superadditivity $\mu(\bar{A}) \geq \mu\left(A^{o}\right)+\mu(\partial A)$, so for any $\mu$-continuity set $A$ we have $\mu(\partial A)=0$.

We have the following generalizations of Aleksandrov's well-known theorem for weak convergence of measures. (Aleksandrov's theorem is often incorrectly called the "Portmanteau theorem," a usage apparently deliberately started by Billingsley, who in [8] cited a paper of the nonexistent mathematician Jean-Pierre Portmanteau, "published" in a nonexistent issue of the Annals of nonexistent university; see [35, p. 130] and [38, p. 313].) This theorem gives equivalent definitions of weak convergence.

Theorem 2.1 Let $X$ be locally compact, and let $\mu, \mu_{\alpha}$ be finite deficient topological measures. The following are equivalent:

(1) $\int f d \mu_{\alpha} \rightarrow \int f d \mu\left(\right.$ i.e. $\left.\mathcal{R}_{\mu_{\alpha}}(f) \rightarrow \mathcal{R}_{\mu}(f)\right)$ for every $f \in C_{0}^{+}(X)$.

(2) $\lim \inf \mu_{\alpha}(U) \geq \mu(U)$ for any $U \in \mathscr{O}(X)$ and $\lim \sup \mu_{\alpha}(K) \leq \mu(K)$ for any $K \in \mathscr{K}(X)$.

(3) $\mu_{\alpha}(A) \rightarrow \mu(A)$ for any compact or open bounded $\mu$-continuity set $A$. 
(4) If $f \in C_{0}^{+}(X)$, then $R_{2, \mu_{\alpha}, f}(t) \rightarrow R_{2, \mu, f}(t)$ and $R_{1, \mu_{\alpha}, f}(t) \rightarrow R_{1, \mu, f}(t)$ for each point $t$ at which $R_{2, \mu, f}$ is continuous.

Proof (1) $\Rightarrow$ (2). Let $U \in \mathscr{O}(X), K \in \mathscr{K}(X), \epsilon>0$. By part (III) of Remark 1.3, choose $f, g \in C_{c}(X)$ such that suppf $\subseteq U, K \subseteq$ supp $g$ and $\mathcal{R}_{\mu}(f)>$ $v(U)-\epsilon, \quad \mathcal{R}_{\mu}(g)<v(K)+\epsilon$. Choose $\alpha_{0}$ such that $\left|\mathcal{R}_{\mu_{\alpha}}(f)-\mathcal{R}_{\mu}(f)\right|<\epsilon$ and $\left|\mathcal{R}_{\mu_{\alpha}}(g)-\mathcal{R}_{\mu}(g)\right|<\epsilon$ for all $\alpha>\alpha_{0}$. Then,

$$
\begin{aligned}
& \mu_{\alpha}(U) \geq \mathcal{R}_{\mu_{\alpha}}(f)>\mathcal{R}_{\mu}(f)-\epsilon>\mu(U)-2 \epsilon, \\
& \mu_{\alpha}(K) \leq \mathcal{R}_{\mu_{\alpha}}(g)<\mathcal{R}_{\mu}(g)+\epsilon<\mu(K)+2 \epsilon,
\end{aligned}
$$

and it is easy to see that $\lim \inf \mu_{\alpha}(U) \geq \mu(U)$ and $\lim \sup \mu_{\alpha}(K) \leq \mu(K)$.

$(2) \Rightarrow(3)$. We have: $\mu\left(A^{o}\right) \leq \liminf \mu_{\alpha}\left(A^{o}\right) \leq \lim \inf \mu_{\alpha}(A) \leq \lim \sup \mu_{\alpha}(A)$ $\leq \limsup \mu_{\alpha}(\bar{A}) \leq \mu(\bar{A})$. If $A$ is an $\mu$-continuity set (whether $A$ is compact or open bounded), we then see that $\lim \mu_{\alpha}(A)=\mu(A)$.

(3) $\Rightarrow$ (4). If $t$ is a point of continuity of $R_{2, \mu, f}$, then from [15, Lemma 6.3 (III)] it follows that the sets $f^{-1}((t, \infty))$ and $f^{-1}([t, \infty))$ are $\mu$-continuity sets.

$(4) \Rightarrow(1)$. By [15, Lemma 6.3], $R_{2, \mu, f}$ has at most countably many points of discontinuity; with notations as in Remark $1.3, r_{\alpha}(\mathbb{R})=\mu_{\alpha}(X)$ converges to $r(\mathbb{R})$ $=\mu(X)$, and $r_{\alpha} \Longrightarrow r$. The statement follows from formula (2).

If $\mu, \mu_{\alpha}$ are finite topological measures on a compact space $X$, and $\lim \mu_{\alpha}(X)$ $=\mu(X)$, then from part (TM1) of Definition 1.4 it follows that lim inf $\mu_{\alpha}(U) \geq \mu(U)$ for any $U \in \mathscr{O}(X)$ iff $\lim \sup \mu_{\alpha}(D) \leq \mu(D)$ for any $D \in \mathscr{C}(X)$. Therefore, with formula (3) we have the following version of Aleksandrov's theorem:

Theorem 2.2 Let $X$ be compact, and let $\mu, \mu_{\alpha}$ be finite topological measures. TFAE:

(1) $\int f d \mu_{\alpha} \rightarrow \int f d \mu$ (i.e., $\mathcal{R}_{\mu_{\alpha}}(f) \rightarrow \mathcal{R}_{\mu}(f)$ ) for every $f \in C^{+}(X)$.

(2) $\int f d \mu_{\alpha} \rightarrow \int f d \mu\left(\right.$ i.e., $\mathcal{R}_{\mu_{\alpha}}(f) \rightarrow \mathcal{R}_{\mu}(f)$ ) for every $f \in C(X)$.

(3) $\liminf \mu_{\alpha} \geq \mu(U)$ for any $U \in \mathscr{O}(X)$ and $\lim \mu_{\alpha}(X)=\mu(X)$.

(4) $\lim \sup \mu_{\alpha}(D) \leq \mu(D)$ for any $D \in \mathscr{C}(X)$ and $\lim \mu_{\alpha}(X)=\mu(X)$.

(5) $\mu_{\alpha}(A) \rightarrow \mu(A)$ for any $\mu$-continuity set $A$.

(6) If $f \in C^{+}(X)$ then $R_{2, \mu_{\alpha}, f}(t) \rightarrow R_{2, \mu, f}(t)$ and $R_{1, \mu_{\alpha}, f}(t) \rightarrow R_{1, \mu, f}(t)$ for each point $t$ at which $R_{2, \mu, f}$ is continuous.

Theorem 2.3 The weak topology on DTM(X) is given by basic neighborhoods of the form

$$
\begin{aligned}
& W\left(v, U_{1}, \ldots, U_{n}, C_{1}, \ldots, C_{m}, \epsilon\right) \\
& \quad=\left\{\mu \in D T M: \mu\left(U_{i}\right)>v\left(U_{i}\right)-\epsilon, \mu\left(C_{j}\right)<v\left(C_{j}\right)+\epsilon,\right. \\
& i=1, \ldots, n, j=1, \ldots m\}
\end{aligned}
$$

where $v \in \mathbf{D T M}(X), U_{i} \in \mathscr{O}(X), C_{j} \in \mathscr{K}(X), \epsilon>0, n, m \in \mathbb{N}$.

Proof The weak topology is the topology $\tau_{N}$ given by basic neighborhoods of the form (4). It is easy to see that the sets $W\left(\nu, U_{1}, \ldots, U_{n}, C_{1}, \ldots, C_{m}, \epsilon\right)$ are basic 
neighborhoods for some topology $\tau_{W}$ on $\mathbf{D T M}(X)$. Consider a basic neighborhood $W(\nu, U, C, \epsilon)$. Given $\epsilon>0$, by part (III) of Remark 1.3 choose $f, g \in C_{c}(X)$ such that suppf $\subseteq U, g \geq 1_{K}$ and

$$
\mathcal{R}_{v}(f)>v(U)-\frac{\epsilon}{2}, \quad \mathcal{R}_{v}(g)<v(C)+\frac{\epsilon}{2} .
$$

Let $\mu \in N(v, f, g, \epsilon / 2)$ as in (4). We have:

$$
\begin{aligned}
& \mu(U)>\mathcal{R}_{\mu}(f)>\mathcal{R}_{v}(f)-\frac{\epsilon}{2}>v(U)-\epsilon, \\
& \mu(C) \leq \mathcal{R}_{\mu}(g)<\mathcal{R}_{\nu}(g)+\frac{\epsilon}{2}<\nu(C)+\epsilon .
\end{aligned}
$$

Therefore, $N(v, f, g, \epsilon / 2) \subseteq W(\nu, U, C, \epsilon)$. We see that $\tau_{W} \subseteq \tau_{N}$, i.e., $\tau_{W}$ is a coarser topology than $\tau_{N}$. If $\mu_{\alpha} \rightarrow \mu$ in the topology $\tau_{W}$, then it is easy to see that $\lim \inf \mu_{\alpha}(U) \geq \mu(U)$ for any open set $U$, and that $\lim \sup \mu_{\alpha}(K) \geq \mu(K)$ for any compact set $K$. By Theorem 2.1, $\int f d \mu_{\alpha} \rightarrow \int f d \mu$ for every $f \in C_{0}^{+}(X)$. The weak topology $\tau_{N}$ is the coarsest topology with this property; thus, $\tau_{N}=\tau_{W}$.

Theorem 2.4 For locally compact $X$, the space $\mathbf{D T M}(X)$ is Hausdorff and locally convex, and every set of the form $\{\mu \in \mathbf{D T M}(X): \mu(X) \leq c\}=\{\mathcal{R}:\|\mathcal{R}\| \leq c\}, c>$ 0 is compact. If $X$ is compact, then $\mathbf{D T M}(X)$ is also locally compact.

Proof First, we shall show that $\operatorname{DTM}(X)$ is Hausdorff. Suppose $\mu \neq v$, then there is $K \in \mathscr{K}(X)$ such that $v(K) \neq \mu(K)$. Let $|\mu(K)-v(K)|=5 \epsilon>0$. By part (III) of Remark 1.3, find $g, h \in C_{c}(X)$ such that $\mathcal{R}_{\mu}(g)-\mu(K)<\epsilon, \mathcal{R}_{v}(h)-v(K)<\epsilon$. Let $f=g \wedge h$, so $\mathcal{R}_{\mu}(f)-\mu(K)<\epsilon, \mathcal{R}_{v}(f)-v(K)<\epsilon$. Then, $N(\mu, f, \epsilon)$ and $N(v, f, \epsilon)$ as in formula (4) are disjoint neighborhoods of $\mu$ and $v$ : Otherwise, if $\lambda \in N(\mu, f, \epsilon) \cap N(v, f, \epsilon)$, then $|\mu(K)-v(K)| \leq\left|\mu(K)-\mathcal{R}_{\mu}(f)\right|+\mid \mathcal{R}_{\mu}(f)-$ $\mathcal{R}_{\lambda}(f)|+| \mathcal{R}_{\lambda}(f)-\mathcal{R}_{v}(f)|+| \mathcal{R}_{v}(f)-v(K)|<4 \epsilon<| \mu(K)-v(K) \mid$, which is a contradiction.

One can also see that DTM $(X)$ is Hausdorff because a homeomorphic space $\boldsymbol{\Phi}^{+}\left(C_{0}^{+}(X)\right)$ is Hausdorff. The basic open set in $\boldsymbol{\Phi}^{+}\left(C_{0}^{+}(X)\right)$ is of the form $W=$ $\left\{\mathcal{R}: \mathcal{R}\left(f_{i}\right) \in O_{i}, O_{i}\right.$ are open in $\left.\mathbb{R}, f_{i} \in C_{0}^{+}(X), i=1, \ldots, n,\right\}$. If $\mathcal{R}$ and $\rho$ are in $W$, then their convex combination is also in $W$. Thus, DTM $(X)$ is locally convex.

Let $c>0$ and $P=\{\mu \in \mathbf{D T M}(X): \mu(X) \leq c\}$. Consider the product space

$$
Y=\prod_{f \in C_{0}^{+}(X)}[-c\|f\|, c\|f\|]
$$

and the function $T: P \longrightarrow Y$ defined by $(T(\mu))_{f}=\rho_{\mu}(f)=\int f d \mu$. The function $T$ is continuous, since each of the maps $\mu \longmapsto \rho_{\mu}(f)$ is continuous. $T$ is $1-1$ which follows from Remark 1.3. Also, $T: P \longrightarrow T(P)$ is a homeomorphism, because $T\left(\mu_{\gamma}\right) \longrightarrow T\left(\mu_{0}\right)$ implies $\mu_{\gamma} \longrightarrow \mu_{0}$. To show that $P$ is compact, it is enough to show that $T(P)$ is closed in $Y$. Let $T\left(\mu_{\alpha}\right) \longrightarrow L$ in $Y$. Define $\rho(f)=L_{f}, f \in$ $C_{0}^{+}(X)$. Then, $\rho$ is a p-conic quasi-linear functional, and by Remark 1.3, there exists 
a finite deficient topological measure $\mu_{0}$ such that $\rho=\rho_{\mu_{0}}$. Then, $L_{f}=\rho_{f}=$ $\left(\rho_{\mu_{0}}\right)_{f}=\left(T\left(\mu_{0}\right)\right)_{f}$, i.e., $L=T\left(\mu_{0}\right)$.

If $X$ is compact, then for $v \in \mathbf{D T M}(X)$ and $W=\{\mu: \mu(X)<v(X)+\epsilon\}$ we have: $v \in W \subseteq\{\mu: \mu(X) \leq v(X)+\epsilon\}$, and the last set is compact.

\section{Prokhorov's Theorem for Topological Measures}

In this section, we show that several classical results of probability theory hold for deficient topological measures or topological measures.

Lemma 3.1 If each sequence $\left\{\mu_{n_{i}}\right\}$ of $\left\{\mu_{n}\right\}$, where $\mu_{n}$ are deficient topological measures, contains a further subsequence $\left\{\mu_{n_{i_{j}}}\right\}$ such that $\mu_{n_{i_{j}}}$ converges weakly to a deficient topological measure $\mu$, then $\mu_{n}$ converges weakly to $\mu$.

Proof If $\mu_{n}$ does not converge weakly to $\mu$, then there is $f \in C_{0}^{+}(X)$ such that $\left|\int f d \mu_{n_{i}}-\int f d \mu\right| \geq \epsilon$ for some $\epsilon>0$ and all $\mu_{n_{i}}$ in some subsequence. But then no subsequence of $\left\{\mu_{n_{i}}\right\}$ can converge weakly to $\mu$.

We clearly have

Lemma 3.2 $X$ is homeomorphic to the (topological) subset $D=\left\{\delta_{x}: x \in X\right\}$ of DTM $(X)$ (equipped with the weak topology).

Theorem 3.1 Let $c \geq 0$. Then, $P=\{\mu \in \mathbf{D T M}(X): \mu(X) \leq c\}$ can be metrized as a separable metric space iff $X$ is a separable metric space.

Proof Suppose $X$ is a separable metric space. By Urysohn's metrization theorem (see [30, p. 125]), $X$ can be topologically embedded in a countable product of unit intervals. Consequently, there exists an equivalent totally bounded metrization on $X$. We will consider this metric on $X$. From [34, Lemma 6.3], $C_{b}(X)$ is separable. Let $\left\{f_{1}, f_{2}, \ldots\right\}$ be a countable dense subset of $C_{b}(X)$.

Let $Y$ be a countable product of $\mathbb{R}$. Define a map $T: P \longrightarrow Y$ as in Theorem 2.4, i.e., $T(\mu)=\left(\int f_{1} d \mu, \int f_{2} d \mu, \ldots\right)$. We will show that $T$ is a homeomorphism on $P$. First, $T$ is $1-1$. (If $T(\mu)=T(\nu)$, then $\int f_{i} d \mu=\int f_{i} d v$ for all $i$, and, hence, $\int f d \mu=\int f d \nu$ for all $f \in C_{0}^{+}(X)$. By Remark, $1.3 \mu=\nu$.) Second, $T$ and $T^{-1}$ are continuous, as in the proof of Theorem 2.4. Since $Y$ is a separable metric space, and $P$ is homeomorphic to a subset of $Y$, it follows that $P$ is a separable metric space.

Conversely, suppose $P$ is a separable metric space. By Lemma 3.2, $X$ is homeomorphic to $D=\left\{\delta_{x}: x \in X\right\}$. $D$ is a separable metric space, and then, so is $X$.

Definition 3.1 Let $X$ be locally compact. A family $\mathcal{M} \subseteq \mathbf{D T M}(X)$ is uniformly tight if for every $\epsilon>0$ there exists a compact set $K_{\epsilon}$ such that $\mu\left(X \backslash K_{\epsilon}\right) \leq \epsilon$ for each $\mu \in \mathcal{M}$. A family $\mathcal{M} \subseteq \mathbf{D T M}(X)$ is uniformly bounded in variation if there is a positive constant $M$ such that $\|\mu\| \leq M$ for each $\mu \in \mathcal{M}$.

One uniformly bounded in variation family that is the often used is the collection of all normalized (i.e., satisfying condition $\mu(X)=1$ ) topological measures on a compact space. 
Proposition 3.1 Suppose $X$ is locally compact. If a sequence $\left(\mu_{n}\right) \in \mathbf{D T M}(X)$ is weakly fundamental (i.e., $\int f d \mu_{n}$ is a fundamental sequence for each $f \in C_{0}^{+}(X)$ ), then it is uniformly bounded in variation.

Proof If not, then there is a subsequence $\left(\mu_{n_{k}}\right)$ such that $\left\|\mu_{n_{k}}\right\|>k 2^{k}$ for each $k$, and by part (III) of Remark 1.3, there are functions $f_{n_{k}} \in C_{c}(X), 0 \leq f_{n_{k}} \leq 1$ such that $\int_{X} f_{n_{k}} d \mu_{n_{k}}>k 2^{k}$. Then, the function $f=\sum_{k=1}^{\infty} \frac{f_{n_{k}}}{2^{k}} \in C_{0}^{+}(X), 0 \leq f \leq 1$, and $\int_{X} f d \mu_{n_{k}} \geq k$ for each $k$. This contradicts the fact that the sequence $\left(\int f d \mu_{n}\right)$ is Cauchy and, hence, bounded.

Theorem 3.2 Suppose $\mathcal{M} \subseteq \mathbf{D T M}(X)$ is a family of finite deficient topological measures such that every sequence in $\mathcal{M}$ contains a weakly convergent subsequence. Then, $\mathcal{M}$ is uniformly bounded in variation.

Proof If not, then there is a sequence $\mu_{n} \subseteq \mathcal{M}$ such that $\left\|\mu_{n}\right\|>n$ for every natural $n$. Let $m_{n_{k}}$ be its weakly convergent subsequence. Then, $\left\|m_{n_{k}}\right\|>n_{k}$, while by Proposition 3.1, this subsequence must be uniformly bounded in variation.

Theorem 3.3 Suppose $X$ is locally compact. Suppose $\mathcal{M} \subseteq \mathbf{T M}(X)$ is a family of finite topological measures such that every sequence in $\mathcal{M}$ contains a weakly convergent subsequence. Then, $\mathcal{M}$ is uniformly tight.

Proof Suppose $\mathcal{M}$ is not uniformly tight. Then, there exists $\epsilon>0$ such that for every compact $K$, one can find $\mu^{K} \in \mathcal{M}$ with

$$
\mu^{K}(X \backslash K)>\epsilon .
$$

Take $\mu_{1}$ to be any topological measure with $\left\|\mu_{1}\right\|>\epsilon$, and let $K_{1} \in \mathscr{K}(X)$ be such that $\mu_{1}\left(K_{1}\right)>\epsilon$. Then, by Lemma 1.1 there is $V_{1} \in \mathscr{O}(X)$ with compact closure such that $K_{1} \subseteq V_{1}$ and so $\mu_{1}\left(\overline{V_{1}}\right)>\epsilon$. By (5), find $\mu_{2}$ satisfying $\mu_{2}\left(X \backslash \overline{V_{1}}\right)>\epsilon$, and let $K_{2} \in \mathscr{K}(X)$ be such that $K_{2} \subseteq X \backslash \overline{V_{1}}$ and $\mu_{2}\left(K_{2}\right)>\epsilon$. Find $V_{2} \in \mathscr{O}(X)$ with compact closure such that $K_{2} \subseteq V_{2} \subseteq \overline{V_{2}} \subseteq X \backslash \overline{V_{1}}$, so $\mu_{2}\left(\overline{V_{2}}\right)>\epsilon$. Find a topological measure $\mu_{3}$ with $\mu_{3}\left(X \backslash\left(\overline{V_{1}} \sqcup \overline{V_{2}}\right)>\epsilon\right.$, and so on. By induction, we find a sequence of compact sets $K_{j}$, a sequence of open sets $V_{j}$ with compact closure, and a sequence of topological measures $\mu_{j} \in \mathcal{M}$ with the following properties: $K_{j} \subseteq V_{j} \subseteq \overline{V_{j}}, \overline{V_{j}}$ are pairwise disjoint, and

$$
\mu_{j}\left(\overline{V_{j}}\right) \geq \mu_{j}\left(K_{j}\right)>\epsilon, \quad K_{j+1} \subseteq \overline{V_{j+1}} \subseteq X \backslash \bigsqcup_{i=1}^{j} \overline{V_{i}}
$$

By part (III) of Remark 1.3 find functions $f_{j} \in C_{c}(X), 1_{K} \leq f_{j} \leq 1, \operatorname{suppf}_{j} \subseteq V_{j}$, with

$$
\int_{X} f_{j} \mathrm{~d} \mu_{j}>\epsilon
$$

By our assumption, the sequence $\left(\mu_{j}\right)$ contains a weakly convergent subsequence. For notational simplicity, assume that $\left(\mu_{j}\right)$ itself weakly converges to $\mu$. 
By Theorem 3.2, we may assume that $\mathcal{M}$ is uniformly bounded in variation by $M$. We let

$$
a_{n}^{i}=\int_{X} f_{i} \mathrm{~d} \mu_{n}
$$

Then, $a_{n}:=\left(a_{n}^{1}, a_{n}^{2}, \ldots,\right)$ belongs to $l^{1}$, because for each $m \in \mathbb{N}, f_{1} \cdot f_{2} \cdot \ldots \cdot f_{m}$ $=0, f_{1}+\ldots+f_{m} \in C_{c}(X), 0 \leq f_{1}+\ldots+f_{m} \leq 1$, and so by part (III) of Remark 1.3 each partial sum $\sum_{i=1}^{m} a_{n}^{i}=\int_{X}\left(f_{1}+f_{2}+\ldots+f_{m}\right) d \mu_{n} \leq\left\|\mu_{n}\right\| \leq M$.

Let $\lambda=\left(\lambda_{i}\right) \in l^{\infty}$. Set $g_{k}=\sum_{i=1}^{k} \lambda_{i} f_{i}$ for $k \in \mathbb{N}$. Each $g_{k} \in C_{c}(X), g_{k}^{+}=$ $\sum_{\lambda_{i} \geq 0, i \in\{1, \ldots, k\}} \lambda_{i} f_{i}$, and

$$
\int_{X} g_{k}^{+} \mathrm{d} \mu \leq\|\lambda\|\|\mu\| \leq\|\lambda\| M
$$

The sequence of functions $\left(g_{1}^{+}, g_{2}^{+}, \ldots\right)$ is increasing, so by part (II) of Remark 1.3 the sequence of integrals $\left(\int_{X} g_{k}^{+} d \mu\right)$ is increasing and, hence, converges as $k \rightarrow$ $\infty$. Similarly, the sequence $\left(\int_{X} g_{k}^{-} d \mu\right)$ converges; hence, the sequence $\left(\int_{X} g_{k} d \mu\right)$ converges as $k \rightarrow \infty$. By weak convergence of $\left(\mu_{n}\right)$ to $\mu$

$$
\lim _{n \rightarrow \infty} \int_{X} g_{k} \mathrm{~d} \mu_{n}=\int_{X} g_{k} \mathrm{~d} \mu
$$

so the double sequence of numbers $z_{n m}=\int_{X} g_{k} \mathrm{~d} \mu_{n}$ converges, and then, for the sequence of numbers $c_{n}=\left\langle\lambda, a_{n}\right\rangle=\sum_{i=1}^{\infty} \lambda_{i} a_{n}^{i}$

$$
\lim _{n \rightarrow \infty} c_{n}=\lim _{n \rightarrow \infty} \lim _{k \rightarrow \infty} \int_{X} g_{k} \mathrm{~d} \mu_{n}=\lim _{k \rightarrow \infty} \lim _{n \rightarrow \infty} \int_{X} g_{k} \mathrm{~d} \mu_{n}=\lim _{k \rightarrow \infty} \int_{X} g_{k} \mathrm{~d} \mu
$$

exists. Thus, for any $\lambda=\left(\lambda_{i}\right) \in l^{\infty}$ the sequence $\left(\left\langle\lambda, a_{n}\right\rangle\right)_{n=1}^{\infty}$ converges. By [10, Lemma 1.3.7], the sequence $\left(a_{n}\right)$ converges in $l_{1}$-norm. Then, $\lim _{n \rightarrow \infty} a_{n}^{n}=0$, which contradicts our choice of $f_{n}$.

Lemma 3.3 Let $X$ be locally compact. If $\left(\mu_{n}\right)$ is a weakly fundamental sequence of finite deficient topological measures which is also uniformly bounded in variation, then $\mu_{n}$ converges weakly to some finite deficient topological measure $\mu$.

Proof Consider functional $L$ on $C_{0}^{+}(X)$ defined as $L(f)=\lim _{n} \int_{X} f d \mu_{n}$. It is easy to check that $L$ is a p-conic quasi-linear functional. Say, $\left(\mu_{n}\right)$ is uniformly bounded in variation by $M$. Since $L(f) \leq\left\|\mu_{n}\right\| \leq M$ for any $f \in C_{0}^{+}(X), 0 \leq f \leq 1$, we see that $L \in \boldsymbol{\Phi}^{+}\left(C_{0}^{+}(X)\right)$, and by Remark 1.3, there is a finite deficient topological measure $\mu$ such that $L(f)=\int_{X} f d \mu$.

Theorem 3.4 Suppose $X$ is a locally compact space such that $C_{0}^{+}(X)$ is separable. Then, every uniformly bonded in variation sequence of finite topological measures has a subsequence which is weakly fundamental. 
Proof Suppose $\left(\mu_{n}\right) \in \mathbf{D T M}(X)$ and $\left\|\mu_{n}\right\| \leq M$ for each $n$. Let $g \in C_{0}^{+}(X)$, so $0 \leq g \leq b$ for some $b$. Each of the functions $R_{2, \mu_{n}, g}(t)$ is monotone and bounded above by $M$ on $[0, b]$. By the Helly-Bray theorem (see [10, Theorem 1.4.6]), there is pointwise convergent subsequence $R_{2, \mu_{n_{i}}, g}$. Then, the sequence of integrals $\int_{X} g d \mu_{n_{i}}=\int_{0}^{b} R_{2, \mu_{n_{i}}, g}(t) d t$ converges and, hence, is fundamental.

If $G$ is a countable dense set in $C_{0}^{+}(X)$, we pick a first subsequence of $\left(n_{i}\right)$ such that $\left(\int_{X} g_{1} d \mu_{n_{i}}\right)$ is fundamental for the first function $g_{1} \in G$, then we choose a further subsequence $\left(n_{i_{j}}\right)$ for which $\left(\int_{X} g_{2} d \mu_{n_{i_{j}}}\right)$ is fundamental for the function $g_{2} \in G$, and so on. By diagonal process, we obtain a subsequence of $\left(\mu_{n}\right)$ for which the sequence of integrals is fundamental for each $g \in G$. For notational simplicity, let us assume that $\left(\mu_{n}\right)$ is such a subsequence, i.e., $\left(\int_{X} g d \mu_{n}\right)$ is fundamental for each function $g \in G$.

For arbitrary $f \in C_{0}^{+}(X)$ and $\epsilon>0$, choose $g \in G$ such that $\|f-g\| \leq \epsilon$ and $n_{0}$ such that $\left|\int_{X} g d \mu_{n}-\int_{X} g d \mu_{i}\right|<\epsilon$ for $n, i \geq n_{0}$. Then, using Remark 1.4, we have:

$$
\begin{aligned}
& \left|\int_{X} f \mathrm{~d} \mu_{n}-\int_{X} f \mathrm{~d} \mu_{i}\right| \\
& \leq\left|\int_{X} f \mathrm{~d} \mu_{n}-\int_{X} g \mathrm{~d} \mu_{n}\right|+\left|\int_{X} g \mathrm{~d} \mu_{n}-\int_{X} g \mathrm{~d} \mu_{i}\right|+\left|\int_{X} g \mathrm{~d} \mu_{i}-\int_{X} f \mathrm{~d} \mu_{i}\right| \\
& \leq\|f-g\|\left\|\mu_{n}\right\|+\epsilon+\|f-g\|\left\|\mu_{i}\right\| \leq 2 \epsilon M+\epsilon,
\end{aligned}
$$

and the sequence of integrals $\left(\int_{X} f d \mu_{n}\right)$ is fundamental. Thus, $\left(\mu_{n}\right)$ is weakly fundamental.

Remark 3.1 If $X$ is a locally compact Hausdorff space which is second countable or satisfies any of the other equivalent conditions of [29, Theorem 5.3, p.29], then $\hat{X}$, the Aleksandrov one-point compactification of $X$, is a compact metrizable (hence, a second countable) space. Then, $C(\hat{X})$ is separable, and $C_{0}(X)$ is also separable as a subspace of a separable metric space.

For topological measures, we have the following version of Prokhorov's wellknown theorem.

Theorem 3.5 Suppose $X$ is a locally compact space such that $C_{0}^{+}(X)$ is separable. Suppose $\mathcal{M}$ is a family of finite topological measures on $X$. The following are true statements:

1. If every sequence from $\mathcal{M}$ contains a weakly convergent subsequence, then $\mathcal{M}$ is uniformly tight and uniformly bounded in variation.

2. If $\mathcal{M}$ is uniformly bounded in variation, then every sequence from $\mathcal{M}$ contains a weakly convergent subsequence.

Proof (1) follows from Theorem 3.2 and Theorem 3.3. (2) follows from Theorem 3.4 and Lemma 3.3. 


\section{Prokhorov and Kantorovich-Rubenstein Metrics}

It is clear that $d_{o}(\mu, \nu)=\sup \left\{\left|\int_{X} f d \mu-\int_{X} f d \nu\right|: f \in C_{0}^{+}(X)\right\}$ is a metric on $\operatorname{DTM}(X)$, and the topology induced by this metric is the weak topology.

For the rest of this section, let $(X, d)$ be a locally compact metric space. We shall consider two other metrics on DTM $(X)$.

Let $A^{t}=\{x \in X: d(x, A)<t\}$ for $A \in \mathscr{O}(X) \cup \mathscr{C}(X), A \neq \emptyset$, and $\emptyset^{t}=\emptyset$ for all $t>0$. Each $A^{t}$ is an open set. Consider the Prokhorov metric $d_{\mathrm{P}}$ on $\operatorname{DTM}(X)$ :

$$
\begin{gathered}
d_{\mathrm{P}}(\mu, v)=\inf \left\{t>0: \mu(A) \leq v\left(A^{t}\right)+t, v(A) \leq \mu\left(A^{t}\right)+t,\right. \\
\forall A \in \mathscr{O}(X) \cup \mathscr{K}(X)\} .
\end{gathered}
$$

Taking $t=\|\mu\|+\|\nu\|$, we see that inf is well defined.

Note that if $\mu$ and $\nu$ are Borel measures and $A$ is a Borel set, then we obtain the usual definition of Prokhorov's metric (sometimes also called Lévy-Prokhorov metric).

Lemma $4.1 d_{P}$ is a metric on $\mathbf{D T M}(X)$.

Proof It is clear that $d_{\mathrm{P}} \geq 0$ and $d_{\mathrm{P}}(\mu, v)=d_{\mathrm{P}}(v, \mu)$. For any $A \in \mathscr{O}(X) \cup \mathscr{C}(X)$, we have $\mu(A) \leq \mu\left(A^{t}\right)+t$ for all $t>0$, so $d_{\mathrm{P}}(\mu, \mu)=0$. Suppose $d_{\mathrm{P}}(\mu, v)=0$.Then, there is $t_{n} \searrow 0$ such that $\mu(K) \leq v\left(K^{t_{n}}\right)+t_{n}$ and $v(K) \leq \mu\left(K^{t_{n}}\right)+t_{n}$ for all $K \in \mathscr{K}(X)$. For $K \in \mathscr{K}(X)$ and $\epsilon>0$, choose $U \in \mathscr{O}(X)$ such that $K \subseteq U$ and $v(U)<v(K)+\epsilon$. There exists $r>0$ such that $K^{r} \subseteq U$. Then, for $t_{n}<r$

$$
\mu(K) \leq v\left(K^{t_{n}}\right)+t_{n} \leq v(U)+t_{n} \leq v(K)+\epsilon+t_{n} .
$$

It follows that $\mu(K) \leq v(K)$, and, similarly, $v(K) \leq \mu(K)$. Then, $\mu=v$ on $\mathscr{K}(X)$, so $\mu=v$.

Now, we shall show the triangle inequality. Suppose that for all $A \in \mathscr{O}(X) \cup \mathscr{K}(X)$

$$
\begin{aligned}
& \mu(A) \leq \lambda\left(A^{t}\right)+t, \quad \lambda(A) \leq \mu\left(A^{t}\right)+t, \\
& \lambda(A) \leq v\left(A^{r}\right)+r, \quad v(A) \leq \lambda\left(A^{r}\right)+r .
\end{aligned}
$$

Since $\left(A^{t}\right)^{r} \subseteq A_{t+r}$ and $\left(A^{r}\right)^{t} \subseteq A_{t+r}$, we have:

$$
\mu(A) \leq \lambda\left(A^{t}\right)+t \leq v\left(A^{t}\right)^{r}+t+r \leq v\left(A_{t+r}\right)+t+r
$$

and, similarly, $v(A) \leq \mu\left(A_{t+r}\right)+t+r$. Thus, $d_{\mathrm{P}}(\mu, v) \leq t+r$. It follows that $d_{\mathrm{P}}(\mu, v) \leq d_{\mathrm{P}}(\mu, \lambda)+d_{\mathrm{P}}(\lambda, v)$.

Theorem 4.1 Let $(X, d)$ be a locally compact metric space. Suppose $d_{P}\left(\mu_{\alpha}, \mu\right) \rightarrow 0$ for a net $\left(\mu_{\alpha}\right) ; \mu_{\alpha}, \mu \in \mathbf{D T M}(X)$. Then $\mu_{\alpha} \Longrightarrow \mu$.

Proof Suppose $d_{\mathrm{P}}\left(\mu_{\alpha}, \mu\right) \rightarrow 0$.

Let $K \in \mathscr{K}(X)$ and $\epsilon>0$. Choose $U \in \mathscr{O}(X)$ such that $K \subseteq U$ and $\mu(U)<$ $\mu(K)+\epsilon$. There exists $r>0$ such that $K^{t} \subseteq U$ for all $t \leq r$. For $\bar{\delta}=\min \{r, \epsilon\}$, let 
$\alpha_{0}$ be such that $d_{\mathrm{P}}\left(\mu_{\alpha}, \mu\right)<\delta$ for each $\alpha \geq \alpha_{0}$. Then, for each $\alpha \geq \alpha_{0}$ there exists $t_{\alpha}<\delta$ such that $\mu_{\alpha}(K) \leq \mu\left(K^{t_{\alpha}}\right)+t_{\alpha} \leq \mu(U)+\epsilon \leq \mu(K)+2 \epsilon$. Then,

$$
\lim \sup \mu_{\alpha}(K) \leq \mu(K)+2 \epsilon \text {. }
$$

It follows that $\lim \sup \mu_{\alpha}(K) \leq \mu(K)$.

Now, let $U \in \mathscr{O}(X)$ and $\epsilon>0$. Choose $K \in \mathscr{K}(X)$ such that $K \subseteq U$ and $\mu(K)>\mu(U)-\epsilon$. Let $r, \delta$ and $\alpha_{0}$ be as above. Then, for each $\alpha \geq \alpha_{0}$ there exists $t_{\alpha}<\delta$ such that $\mu(K) \leq \mu_{\alpha}\left(K^{t_{\alpha}}\right)+t_{\alpha} \leq \mu_{\alpha}(U)+\epsilon$. Then,

$$
\lim \inf \mu_{\alpha}(U) \geq \mu(K)-\epsilon \geq \mu(U)-2 \epsilon \text {. }
$$

It follows that $\liminf \mu_{\alpha}(U) \geq \mu(U)$.

By Theorem 2.1, $\mu_{\alpha} \Longrightarrow \mu$.

Let family $\mathcal{M} \subseteq \mathbf{T M}(X)$ be uniformly bounded in variation. We consider the Kantorovich-Rubinstein metric $d_{\mathrm{KR}}$ on $\mathcal{M}$

$$
d_{\mathrm{KR}}(\mu, v)=\sup \left\{\left|\int_{X} f \mathrm{~d} \mu-\int_{X} f \mathrm{~d} \nu\right|: f \in \operatorname{Lip}_{1}(X, d) \cap C_{c}(X),\|f\| \leq 1\right\}
$$

where $\operatorname{Lip}_{1}(X)=\{f: X \rightarrow \mathbb{R}:|f(x)-f(y)| \leq d(x, y) \forall x, y \in X\}$.

Remark 4.1 Our definition is related to the definition of the Kantorovich-Rubinstein metric for Borel measures, which is obtained from the Kantorovich-Rubinstein norm

$$
\|\mu\|_{K R}=\sup \left\{\int_{X} f \mathrm{~d} \mu: f \in \operatorname{Lip}_{1}(X, d),\|f\| \leq 1\right\} .
$$

This metric is sometimes also called the Wasserstein metric $W(\mu, v)$, although there is no author with this name. See [9, pp. 453-454, Comments to Ch.8] for a good note on the history and use of this metric.

Our use of $f \in \operatorname{Lip}_{1}(X, d) \cap C_{c}(X)$ in (6) is dictated, on the one hand, by the relation of (6) to Kantorovich-Rubinstein metric for Borel measures and, on the other hand, by the role of $C_{c}(X)$ in the theory of (p-conic) quasi-linear functionals. Note that by [7, Theorem 2] Lipschitz functions with compact support are dense in $C_{0}(X)$.

Lemma $4.2 d_{K R}$ is a metric on a uniformly bounded in variation family $\mathcal{M}$.

Proof We shall show that $d_{\mathrm{KR}}(\mu, v)=0$ implies $\mu=v$; the remaining properties are obvious. Let $M$ be such that $\|\mu\| \leq M$ for each $\mu \in \mathcal{M}$. Take $f \in C_{0}(X)$. Given $\epsilon>0$, choose a Lipschitz function $g$ with compact support so that $\|f-g\|<\epsilon$. Since $d_{\mathrm{KR}}(\mu, \nu)=0$, we see that $\left|\int_{X} g \mathrm{~d} \mu-\int_{X} g \mathrm{~d} \nu\right|=0$. Using also Remark 1.4, 
we have:

$$
\begin{array}{r}
\left|\int_{X} f \mathrm{~d} \mu-\int_{X} f \mathrm{~d} \nu\right| \\
\leq\left|\int_{X} f \mathrm{~d} \mu-\int_{X} g \mathrm{~d} \mu\right|+\left|\int_{X} g \mathrm{~d} \mu-\int_{X} g \mathrm{~d} \nu\right|+\left|\int_{X} g \mathrm{~d} \nu-\int_{X} f \mathrm{~d} \nu\right| \\
\leq\|f-g\| \mu(X)+\|f-g\| v(X) \leq 2 \epsilon M .
\end{array}
$$

Thus, $\int_{X} f d \mu=\int_{X} f d v$ for every $f \in C_{0}(X)$. By Remark 1.3, $\mu=v$.

Theorem 4.2 Let $X$ be a locally compact metric space. In either of the following situations:

1. a family $\mathcal{M} \subseteq \mathbf{T M}(X)$ is uniformly bounded in variation;

2. given $M>0$, a family $\mathcal{M} \subseteq \mathbf{D T M}(X)$ is the family of deficient topological measures corresponding to functionals $\mathcal{R}$ on $C_{c}^{+}(X)$ with $\|\mathcal{R}\| \leq M$;

if a net $\left(\mu_{\alpha}\right) \in \mathcal{M}, \mu \subseteq \mathcal{M}$, and $d_{K R}\left(\mu_{\alpha}, \mu\right) \rightarrow 0$, then $\mu_{\alpha} \Longrightarrow \mu$.

Proof 1. Let $f \in C_{0}(X)$. Given $\epsilon>0$, choose a Lipschitz function with compact support $g$ so that $\|f-g\|<\epsilon$. Since $\left|\int_{X} g d \mu_{\alpha}-\int_{X} g d \mu\right| \leq d_{\mathrm{KR}}\left(\mu_{\alpha}, \mu\right)\|g\|$, assume $\left|\int_{X} g d \mu_{\alpha}-\int_{X} g d \mu\right| \leq \epsilon$ for all $\alpha \geq \alpha_{0}$. Then, for all $\alpha \geq \alpha_{0}$ using Remark 1.4 we have:

$$
\begin{array}{r}
\left|\int_{X} f \mathrm{~d} \mu_{\alpha}-\int_{X} f \mathrm{~d} \mu\right| \\
\leq\left|\int_{X} f \mathrm{~d} \mu_{\alpha}-\int_{X} g \mathrm{~d} \mu_{\alpha}\right|+\left|\int_{X} g \mathrm{~d} \mu_{\alpha}-\int_{X} g \mathrm{~d} \mu\right|+\left|\int_{X} g \mathrm{~d} \mu-\int_{X} f \mathrm{~d} \mu\right| \\
\leq\|f-g\| \mu_{\alpha}(X)+\epsilon+\|f-g\| \mu(X) \leq 2 \epsilon M+\epsilon,
\end{array}
$$

so $\int_{X} f \mathrm{~d} \mu_{\alpha} \longrightarrow \int_{X} f \mathrm{~d} \mu$. It follows that $\mu_{\alpha} \Longrightarrow \mu$.

2. If a deficient topological measure corresponds to $\mathcal{R}$, then $\|\mu\| \leq M$. Thus, the family $\mathcal{M}$ is uniformly bounded in variation, and we may use the same argument as in the previous part.

Theorem 4.3 Let $X$ be a compact metric space. Given $M>0$, let $\mathcal{M}=\{\mu \in$ $\operatorname{DTM}(X):\|\mu\| \leq M\}$. Then, the topology on $\mathcal{M}$ induced by the metric $d_{K R}$ is the weak topology.

Proof By Theorem 4.2, if a net $\left(\mu_{\alpha}\right)$ converges to $\mu$ in the metric $d_{\mathrm{KR}}$, then it also converges to $\mu$ weakly. For $\mathcal{M}=\{\mu \in \mathbf{T M}(X):\|\mu\| \leq 1\}$ and a slightly different metric, the result was first shown in [21, Proposition 1.10], and our proof of Theorem 4.2 follows the argument in that paper. Because of Remark 1.4 and the fact that the family of functions in (6) is compact by the Arzela-Ascoli theorem, one can basically repeat an argument from [21, Proposition 1.10] to show that the weak convergence of $\left(\mu_{\alpha}\right)$ to $\mu$ implies convergence in the metric $d_{\mathrm{KR}}$. 


\section{Density Theorems}

Definition 5.1 A deficient topological measure $v$ is called proper if from $m \leq v$, where $m$ is a Radon measure, it follows that $m=0$.

Remark 5.1 From [14, Theorem 4.3], it follows that a finite deficient topological measure can be written as a sum of a finite Radon measure and a proper finite deficient topological measure. The sum of two proper deficient topological measures is proper (see [14, Theorem 4.5]).

A finite Radon measure on a compact space is a regular Borel measure, so our definition of a proper deficient topological measure (which is given in [14]) coincides with definitions in papers prior to [14].

In what follows, $p \mathbf{D T M}(X)$ and $p \mathbf{T M}(X)$ denote, respectively, the family of proper finite deficient topological measures and the family of proper finite topological measures.

For a compact space $X$, there is a certain topological characteristic genus. See [5] for more information about genus $g$ of the space. A compact space has genus 0 iff any finite union of disjoint closed solid sets has a connected complement. Intuitively, $X$ does not have holes or loops. In the case, where $X$ is locally path connected, $g=0$ if the fundamental group $\pi_{1}(X)$ is finite (in particular, if $X$ is simply connected). Knudsen [31] was able to show that if $H^{1}(X)=0$, then $g(X)=0$, and in the case of CW complexes, the converse also holds.

Remark 5.2 From Theorem 1.1, it is easy to see that if $\mu, v$ are deficient topological measures, and $v$ is not a topological measure, then $\mu+v$ is a deficient topological measure which is not a topological measure.

Theorem 5.1 1. (Proper simple deficient topological measures that are not topological measures are dense in the set of all point masses $) \Longrightarrow(p \mathbf{D T M}(X) \backslash \mathbf{T M}(X)$ is dense in $\mathbf{M}(X)) \Longleftrightarrow(p \mathbf{D T M}(X) \backslash \mathbf{T M}(X)$ is dense in $\mathbf{D T M}(X) \backslash \mathbf{T M}(X)) \Longrightarrow$ $(p \mathbf{D T M}(X)$ is dense in $\mathbf{D T M}(X)) \Longleftrightarrow(p \mathbf{D T M}(X)$ is dense in $\mathbf{M}(X))$.

2. (Proper simple $\mathbf{T M}(X)$ are dense in the set of all point masses $) \Longrightarrow(p \mathbf{T M}(X)$ is dense in $\mathbf{M}(X)) \Longleftrightarrow(p \mathbf{T M}(X)$ is dense in $\mathbf{T M}(X)) \Longrightarrow(p \mathbf{D T M}(X)$ is dense in $\operatorname{DTM}(X))$.

Proof We shall prove the first part; the proof of the second part is similar, but simpler.

(A) We shall show the first implication. Any measure is approximated by convex combinations of point masses, so by assumption, it is approximated by convex combinations of proper simple deficient topological measures that are not topological measures. By Remarks 5.1 and 5.2, the latter combinations are in $p \mathbf{D T M}(X) \backslash \mathbf{T M}(X)$.

(B) $(p \mathbf{D T M}(X) \backslash \mathbf{T M}(X)$ is dense in $\mathbf{M}(X)) \Longrightarrow(p \mathbf{D T M}(X) \backslash \mathbf{T M}(X)$ is dense in $\mathbf{D T M}(X) \backslash \mathbf{T M}(X))$ : Suppose $\mu \in \mathbf{D T M}(X) \backslash \mathbf{T M}(X)$. By Remark 5.1, write $\mu=m+\mu^{\prime}$, where $\mu^{\prime}$ is a proper deficient topological measure, and $m$ is a measure from $\mathbf{M}(X)$. By assumption, $m$ is approximated by $v \in$ $p \mathbf{D T M}(X) \backslash \mathbf{T M}(X)$. Then, $\mu$ is approximated by $v+\mu^{\prime}$, where by Remarks 5.1 and $5.2 v+\mu^{\prime}$ is in $p \mathbf{D T M}(X) \backslash \mathbf{T M}(X)$. 
(C) $(p \mathbf{D T M}(X) \backslash \mathbf{T M}(X)$ is dense in $\mathbf{D T M}(X) \backslash \mathbf{T M}(X)) \Longrightarrow(p \mathbf{D T M}(X) \backslash \mathbf{T M}(X)$ is dense in $\mathbf{M}(X))$ : Suppose to the contrary that there exists a measure $m \in \mathbf{M}(X)$ and its neighborhood $N$ which contains no elements of $p \mathbf{D T M}(X) \backslash p \mathbf{T M}(X)$. Take $\lambda \in \mathbf{D T M}(X) \backslash \mathbf{T M}(X)$. Then, for any deficient topological measure $v \in N$ we see that $\lambda+v$ is a deficient topological measure that is not a topological measure and is not proper. Thus, a neighborhood $\lambda+N \subseteq \mathbf{D T M}(X) \backslash \mathbf{T M}(X)$ contains no elements of $p \mathbf{D T M}(X) \backslash \mathbf{T M}(X)$, which contradicts the assumption.

(D) $(p \mathbf{D T M}(X) \backslash \mathbf{T M}(X)$ is dense in $\mathbf{M}(X)) \Longrightarrow(p \mathbf{D T M}(X)$ is dense in DTM $(X))$ : Let $\mu \in \mathbf{D T M}(X)$. Write again $\mu=m+\mu^{\prime}$, where $m \in \mathbf{M}(X)$ and $\mu^{\prime} \in$ $p \mathbf{D T M}(X)$. By assumption, $m$ is approximated by some $\lambda \in p \mathbf{D T M}(X)$. Then, $\lambda+\mu^{\prime} \in p \mathbf{D T M}(X)$ and approximates $\mu$.

(E) $(p \mathbf{D T M}(X)$ is dense in $\mathbf{D T M}(X)) \Longrightarrow(p \mathbf{D T M}(X)$ is dense in $\mathbf{M}(X))$ : obvious.

(F) $(p \mathbf{D T M}(X)$ is dense in $\mathbf{M}(X)) \Longrightarrow(p \mathbf{D T M}(X)$ is dense in DTM $(X))$ : follows from Remarks 5.1 and 5.2 in a manner similar to the one in part (B).

Theorem 5.2 Suppose any open set in a locally compact space X contains a compact connected subset that is not a singleton. Then, $p \mathbf{D T M}(X)$ is dense in $\mathbf{D T M}(X)$.

Proof If we shall show that proper simple DTM $(X) \backslash \mathbf{T M}(X)$ are dense in the set of point masses, then the statement will follow from Theorem 5.1. Let $\delta_{a}$ be a point mass at $a$. Let $\{V \in \mathscr{O}(X): a \in V\}$ be ordered by reverse inclusion. For each $V$, let $K_{V} \subseteq V$ be the non-singleton connected compact set. Consider $\lambda^{V}$ defined on $\mathscr{O}(X) \cup \mathscr{C}(X)$ as follows: $\lambda^{V}(A)=1$ if $K_{V} \subseteq A$ and $\lambda^{V}(A)=0$ otherwise. By Example 1.3, $\lambda^{V}$ is simple and $\lambda^{V} \in \mathbf{D T M}(X) \backslash \mathbf{T M}(X)$. If $U \in \mathscr{O}(X)$ and $\delta_{a}(U)=1$, then $a \in U$ and for all $V \subseteq U, V \in \mathscr{O}(X)$ we have $K_{V} \subseteq U$, so $\lambda^{V}(U)=1$. Then, $\liminf \lambda^{V}(U)=1=\delta_{a}(U)$. If $C \in \mathscr{K}(X)$ and $\delta_{a}(C)=0$, then $a \notin C$ and we may find $U \in \mathscr{O}(X)$ such that $a \in U, U \cap C=\emptyset$. Then, for each $V \subseteq U, V \in \mathscr{O}(X)$ we have $K_{V} \cap C=\emptyset$ and $\lambda^{V}(C)=0$. Then, $\lim \sup \lambda^{V}(C)=0=\delta_{a}(C)$. By Theorem 2.1, the net $\left(\lambda^{V}\right)$ converges weakly to $\delta_{a}$.

Remark 5.3 Among spaces that satisfy the condition of the previous theorem are: non-singleton locally compact spaces that are locally connected or weakly locally connected; manifolds; CW complexes.

Theorem 5.3 Suppose X is a non-singleton connected, locally connected, locally compact space with no cut points and such that the Aleksandrov one-point compactification of $X$ has genus 0 . Then, $p \mathbf{T M}(X)$ is dense in $\mathbf{T M}(X)$, and $p \mathbf{D T M}(X)$ is dense in $\operatorname{DTM}(X)$.

Proof We shall give the proof for the case when $X$ is not compact. (When $X$ is compact, the proof is similar but simpler; also, one may use [41, Theorem 4.9].) We shall show that proper simple topological measures are dense in the set of simple measures, and the statements will follow from part (2) of Theorem 5.1.

Let $\delta_{a}$ be a point mass. It is enough to show that a neighborhood of the form $W\left(\delta_{a}, U, C, \epsilon\right)$ as in Theorem 2.3 contains a simple proper topological measure.

Suppose first $a \in U \in \mathscr{O}(X), a \notin C$. We may assume that $U \cap C=\emptyset$. Since $a \in U \in \mathscr{O}(X)$, by Lemma 1.1 there is a bounded open connected set $V$ and a 
compact connected set $D$ such that $a \in V \subseteq D \subseteq U$. Since $X$ is connected and non-singleton, $a \subsetneq V$, and we may choose 3 different points in $D$. Let $\lambda$ be a simple topological measure on $X$ given by [19, Example 15.9], so (similar to Example 1.2) $\lambda(A)=1$ if a bounded solid set $A$ contains two or three of the chosen points, and $\lambda(A)=0$ if a bounded solid set $A$ contains no more than one of the chosen points. Since the solid hull of $D$ (a compact solid set) contains all three points, and each bounded component of $X \backslash D$ (a bounded open solid set) contains none of the three points, by $[19$, Definition 7.1] we compute $\lambda(D)=1$. Then $\lambda(U)=1$. Since $C$ is disjoint from $U$, and $\lambda(X)=1$, by superadditivity we have $\lambda(C)=0$. Thus, $\lambda \in W\left(\delta_{a}, U, C, \epsilon\right)$.

We shall show that $\lambda$ is proper. Let $x \in X$. Since $X \backslash\{x\}$ is connected, by Lemma 1.1 there is a compact connected set $B \subseteq X \backslash\{x\}$ such that $B$ contains at least two of the three chosen points. Argument as above shows that $\lambda(B)=1$. Then, $\lambda(\{x\})$ $\leq \lambda(X \backslash B)=\lambda(X)-\lambda(B)=0$. Thus, $\lambda(\{x\})=0$ for any $x \in X$, and by [14, Lemma 4.12], $\lambda$ is proper.

The remaining three cases are easy. For example, if $a \in U, a \in C$, then $\lambda$ as above will do.

Lemma 5.1 Suppose $X$ is locally compact, $\sum_{i=1}^{\infty} \mu_{i}(X)<\infty$ where each $\mu_{i}$ is a deficient topological measure. Then, $\mu=\sum_{i=1}^{\infty} \mu_{i}$ is a finite deficient topological measure. If each $\mu_{i}$ is a topological measure, then $\mu$ is a finite topological measure.

Proof Let $\mu=\sum_{i=1}^{\infty} \mu_{i}$ on $\mathscr{O}(X) \cup \mathscr{C}(X)$. It is easy to see that $\mu$ is finitely additive on compact sets. For $\epsilon>0$, let $j$ be such that $\sum_{i=j+1}^{\infty} \mu_{i}(X)<\epsilon$, and let $\lambda=$ $\sum_{i=1}^{j} \mu_{i}$. Then, $\lambda$ is a finite deficient topological measure. For $U \in \mathscr{O}(X)$, there exists $K \in \mathscr{K}(X)$ such that $\lambda(U)<\lambda(K)+\epsilon$. Then, $\mu(U)<\lambda(U)+\epsilon<\lambda(K)+2 \epsilon$ $<\mu(K)+2 \epsilon$, and the inner regularity of $\mu$ follows. Similarly, $\mu$ is outer regular. Thus, $\mu$ is a deficient topological measure; clearly, $\mu$ is finite. If each $\mu_{i}$ is a topological measure, it is easy to check additivity of $\mu$ on $\mathscr{O}(X) \cup \mathscr{K}(X)$, so condition (TM1) of Definition 1.4 holds, and $\mu$ is a topological measure.

Lemma 5.2 Suppose $X$ is locally compact, $\sum_{i=1}^{\infty} \mu_{i}(X)<\infty$ where each $\mu_{i}$ is a proper deficient topological measure (respectively, a proper topological measure). Then, $\mu=\sum_{i=1}^{\infty} \mu_{i}$ is a finite proper deficient topological measure (respectively, $a$ finite proper topological measure).

Proof By Lemma 5.1, $\mu$ is a finite deficient topological measure (respectively, a finite topological measure). We need to show that $\mu$ is proper. By Remark 5.1, write $\mu$ $=m+\mu^{\prime}$, where $m$ is a finite Radon measure and $\mu^{\prime}$ is a proper deficient topological measure. We shall show that $m=0$.

Let $K \in \mathscr{K}(X)$. For $\epsilon>0$, let $N$ be such that $\sum_{i=N+1}^{\infty} \mu_{i}(X)<\epsilon$, and let $\mu^{N}=\sum_{i=1}^{N} \mu_{i}$.

By Remark 5.1, $\mu^{N}$ is a proper deficient topological measure. By [14, Theorem 4.4], there are compact sets $K_{1}, \ldots, K_{n}$ such that $K=\cup K_{j}$ and $\sum_{j=1}^{n} \mu^{N}\left(K_{j}\right)<\epsilon$. Let $E_{1}, \ldots, E_{n}$ be disjoint Borel sets such that $E_{j} \subseteq K_{j}$ and $\bigsqcup_{j=1}^{n} E_{i}=\bigcup_{j=1}^{n} K_{j}$. Since $m$ is finite, outer regularity of $m$ is equivalent to inner closed regularity of $m$. Find disjoint sets $C_{j}, C_{j} \subseteq E_{j} \subseteq K_{j}, j=1, \ldots, n$ such that $C_{j}$ are closed (hence, 
compact) and $m\left(C_{j}\right)>m\left(E_{j}\right)-\frac{\epsilon}{n}$. Then,

$$
\begin{aligned}
m(K)= & \sum_{j=1}^{n} m\left(E_{j}\right) \leq \epsilon+\sum_{j=1}^{n} m\left(C_{i}\right) \leq \epsilon+\mu\left(C_{1} \sqcup \ldots \sqcup C_{n}\right) \\
& \leq \epsilon+\mu^{N}\left(C_{1} \sqcup \ldots \sqcup C_{n}\right)+\epsilon=2 \epsilon+\sum_{j=1}^{n} \mu^{N}\left(C_{j}\right) \\
& \leq 2 \epsilon+\sum_{j=1}^{n} \mu^{N}\left(K_{i}\right) \leq 3 \epsilon .
\end{aligned}
$$

It follows that $m(K)=0$ for any $K \in \mathscr{K}(X)$. Thus, $m=0$, and $\mu$ is proper.

Theorem 5.4 Let $X$ be locally compact. Suppose $X=\bigcup_{i=1}^{\infty} X_{i}$, where each $X_{i}$ is a compact subset of $X$.

1. If $p \mathbf{D T M}\left(X_{i}\right)$ is dense in $\mathbf{M}\left(X_{i}\right), i \in \mathbb{N}$, then $p \mathbf{D T M}(X)$ is dense in $\mathbf{M}(X)$.

2. If $p \mathbf{T M}\left(X_{i}\right)$ is dense in $\mathbf{M}\left(X_{i}\right), i \in \mathbb{N}$, then $p \mathbf{T} \mathbf{M}(X)$ is dense in $\mathbf{M}(X)$.

Proof Note that each $X_{i}$ is a locally compact space with respect to the subspace topology. We shall prove the first part. Let $m \in \mathbf{M}(X)$. We shall show that every neighborhood $W$ of $m$ as in Theorem 2.3 contains a proper deficient topological measure. To simplify notation, we consider $W(m, U, C, \epsilon)$ where $U \in \mathscr{O}(X), C \in$ $\mathscr{K}(X), \epsilon>0$. Take Borel subsets $Y_{i}$ of $X$ such that $Y_{i} \subseteq X_{i}$ and $\bigsqcup_{i=1}^{\infty} Y_{i}=X$. Consider $m_{i}(B)=m\left(B \cap Y_{i}\right)$, where $B$ is a Borel set in $X_{i}, i \in \mathbb{N}$. It is easy to see that $m_{i} \in \mathbf{M}\left(X_{i}\right)$.

Let $\epsilon>0$. Let $U_{i}=U \cap X_{i}, C_{i}=C \cap X_{i}, \epsilon_{i}=\epsilon 2^{-i}$ for $i \in \mathbb{N}$, so $U_{i}$ is open in $X_{i}$ and $C_{i}$ is compact in $X_{i}$. By assumption, there is $\lambda_{i} \in p \operatorname{DTM}\left(X_{i}\right)$ such that $\lambda_{i} \in W\left(m_{i} ; U_{i}, C_{i}, X_{i}, \epsilon_{i}\right)$. Let $v_{i}$ be the extension of $\lambda_{i}$ to $\mathscr{O}(X) \cup \mathscr{C}(X)$ given by $v_{i}(A)=\lambda_{i}\left(A \cap X_{i}\right)$ for $A \in \mathscr{O}(X) \cup \mathscr{C}(X)$. It is easy to see that $v_{i}$ is a deficient topological measure, and $v_{i}(X)=\lambda_{i}\left(X_{i}\right)<\infty$. Since $\lambda_{i}$ is proper, by [14, Theorem 4.4] given $\delta>0$ there are sets of the form $V_{j} \cap X_{i}, V_{j} \in \mathscr{O}(X), j=1, \ldots, n$ such that they cover $X_{i}$ and $\sum_{j=1}^{n} \lambda_{i}\left(V_{j} \cap X_{i}\right)<\delta$. Then, open sets $V_{1}, \ldots, V_{n}, X \backslash X_{i}$ cover $X$ and $\sum_{j=1}^{n} v_{i}\left(V_{j}\right)+v_{i}\left(X \backslash X_{i}\right)=\sum_{j=1}^{n} \lambda_{i}\left(V_{j} \cap X_{i}\right)<\delta$, and so $v_{i} \in p \mathbf{D T M}(X)$ by [14, Theorem 4.4].

Since $\sum_{i=1}^{\infty} v_{i}(X)=\sum_{i=1}^{\infty} \lambda_{i}\left(X_{i}\right) \leq \sum_{i=1}^{\infty}\left(m_{i}\left(X_{i}\right)+\epsilon_{i}\right)=m(X)+\epsilon<\infty$, by Lemma $5.2 v=\sum_{i=1}^{\infty} v_{i}$ is a finite proper deficient topological measure. We have:

$$
\begin{aligned}
& \nu(U)=\sum_{i=1}^{\infty} v_{i}(U)=\sum_{i=1}^{\infty} \lambda_{i}\left(U \cap X_{i}\right)>\sum_{i=1}^{\infty}\left(m_{i}\left(U \cap X_{i}\right)-\epsilon_{i}\right)=m(U)-\epsilon, \\
& \nu(C)=\sum_{i=1}^{\infty} v_{i}(C)=\sum_{i=1}^{\infty} \lambda_{i}\left(C \cap X_{i}\right)<\sum_{i=1}^{\infty}\left(m_{i}\left(C \cap X_{i}\right)+\epsilon_{i}\right)=m(C)+\epsilon .
\end{aligned}
$$

Thus, $v \in W(m, U, C, \epsilon)$. 
The proof of the second part is the same, taking into account that $\lambda_{i}, v_{i}, v$ are proper topological measures.

Corollary 5.1 Let $X=\cup_{i=1}^{\infty} X_{i}$, where each $X_{i}$ as in Theorem 5.3. Then, $p \mathbf{T M}(X)$ is dense in $\mathbf{T M}(X)$, and $p \mathbf{D T M}(X)$ is dense in $\mathbf{D T M}(X)$.

Proof By part 2 of Theorem 5.1, it is enough to show that $p \mathbf{T M}(X)$ is dense in $\mathbf{M}(X)$. By Theorem 5.3, $p \mathbf{T M}\left(X_{i}\right)$ is dense in $\mathbf{M}\left(X_{i}\right)$ for each $i$, and we apply part 2 of Theorem 5.4.

Remark 5.4 In Corollary 5.1, one may take, for example, a compact n-manifold, $n \geq 2$ as $X$, or $X$ that is covered by countably many sets homeomorphic to balls $B^{n}$ with varying $n \geq 2$.

Lemma 5.3 $\mathbf{T M}(X)$ is a closed subset of $\mathbf{D T M}(X)$, and $\mathbf{M}(X)$ is a closed subset of $\operatorname{DTM}(X)$.

Proof By Remark 1.3, $\mu \in \mathbf{T M}(X)$ iff $\rho$ is a quasi-linear functional on $C_{0}(X)$, and $\mu \in \mathbf{M}(X)$ iff $\rho$ is a linear functional on $C_{0}(X)$, where $\rho(f)=\mathcal{R}_{\mu}\left(f^{+}\right)-\mathcal{R}_{\mu}\left(f^{-}\right)$. Using basic open sets in Definition 2.1, it is easy to check that $\mathbf{T M}(X)$ is a closed subset of DTM $(X)$, and $\mathbf{M}(X)$ is a closed subset of DTM $(X)$.

Theorem 5.5 Suppose $X$ is locally compact. The following are equivalent:

1. $\mathbf{M}(X)$ is nowhere dense in $\mathbf{D T M}(X)$ (respectively, in $\mathbf{T M}(X)$ ).

2. There exists a finite deficient topological measure (respectively, a finite topological measure) that is not a measure.

3. There exists a nonzero finite proper deficient topological measure (respectively, nonzero finite proper topological measure).

Proof (1) $\Longrightarrow$ (2) is obvious. (2) $\Longrightarrow$ (3): Let $\mu$ be a deficient topological measure that is not a measure. By Remark 5.1, write $\mu=m+\mu^{\prime}$ where $m$ is a measure and $\mu^{\prime}$ is a proper deficient topological measure. Then, $\mu^{\prime} \neq 0$. (3) $\Longrightarrow(1)$ : Suppose $v \neq 0$ is a proper finite deficient topological measure. Let $m \in \mathbf{M}(X)$. Consider a set function $\mu_{n}$ on $\mathscr{O}(X) \cup \mathscr{C}(X)$ given by

$$
\mu_{n}(A)=\frac{1}{n} \frac{1}{v(X)} v(A)+\left(1-\frac{1}{n}\right) m(A) .
$$

Then, each $\mu_{n}$ is a deficient topological measure that is not a measure, and $\mu_{n} \Longrightarrow m$ by Theorem 2.1. Thus, DTM $(X) \backslash \mathbf{M}(X)$ is dense in $\mathbf{M}(X)$, and since $\mathbf{M}(X)$ is a closed subset of DTM $(X)$, we see that $\mathbf{M}(X)$ is nowhere dense in $\mathbf{D T M}(X)$. The proof for topological measures is similar.

Corollary 5.2 Suppose $X$ is locally compact. If $X$ contains a non-singleton compact connected set, then $\mathbf{M}(X)$ is nowhere dense in $\mathbf{D T M}(X)$. If $X$ contains an open (or closed) locally connected, connected, non-singleton subset whose Aleksandrov onepoint compactification has genus 0 , then $\mathbf{M}(X)$ is nowhere dense in $\mathbf{T M}(X)$. 
Proof Use part (2) of Theorem 5.5. For the first statement, Example 1.3 gives a finite deficient topological measure that is not a topological measure (hence, not a measure). For the second statement, as an example of a finite topological measure that is not a measure one may take [19, Example 15.5].

The proof of the next theorem and corollary is similar to the proof of Theorem 5.5 and Corollary 5.2.

Theorem 5.6 Suppose $X$ is locally compact. The following are equivalent:

\section{1. $\mathbf{T M}(X)$ is nowhere dense in $\mathbf{D T M}(X)$.}

2. There exists a finite deficient topological measure that is not a topological measure.

3. There exists a nonzero finite proper deficient topological measure that is not a topological measure.

Corollary 5.3 If a locally compact space X contains a non-singleton compact connected set, then $\mathbf{T M}(X)$ is nowhere dense in $\mathbf{D T M}(X)$.

Remark 5.5 When the space is compact, the equivalence of the first two conditions in Theorem 2.1 and of conditions (2)-(4) in Theorem 2.2 was first given in [41, Corollary $4.4,4.5]$. When $X$ is compact, Theorem 2.3 was proved in [41], but the method there does not work for a locally compact non-compact space, as the set $f^{-1}([0, \infty))=X$ is not compact. Theorem 2.4 generalizes results from several papers, including [2,26], and [41]. Theorem 3.1 is an adaptation of [34, Theorem 6.2]. Our proof of Theorem 3.3 is adapted from a nice proof in [10, Theorem 2.3.4]. In the last section, we generalize results from [41, Sect. 4] and [11] from a compact space to a locally compact one.

Acknowledgements The author would like to thank the Department of Mathematics at the University of California, Santa Barbara, for its supportive environment.

\section{Declarations}

Conflict of interest The author declares no conflict of interest.

Open Access This article is licensed under a Creative Commons Attribution 4.0 International License, which permits use, sharing, adaptation, distribution and reproduction in any medium or format, as long as you give appropriate credit to the original author(s) and the source, provide a link to the Creative Commons licence, and indicate if changes were made. The images or other third party material in this article are included in the article's Creative Commons licence, unless indicated otherwise in a credit line to the material. If material is not included in the article's Creative Commons licence and your intended use is not permitted by statutory regulation or exceeds the permitted use, you will need to obtain permission directly from the copyright holder. To view a copy of this licence, visit http://creativecommons.org/licenses/by/4.0/.

\section{References}

1. Aarnes, J.F.: Physical States on $C^{*}$-algebra. Acta Math. 122, 161-172 (1969)

2. Aarnes, J.F.: Quasi-states on $C^{*}$-algebras. Trans. Amer. Math. Soc. 149, 601-625 (1970)

3. Aarnes, J.F.: Quasi-states and quasi-measures. Adv. Math. 86(1), 41-67 (1991)

4. Aarnes, J.F.: Pure quasi-states and extremal quasi-measures. Math. Ann. 295, 575-588 (1993) 
5. Aarnes, J.F.: Construction of non-subadditive measures and discretization of Borel measures. Fund. Math. 147, 213-237 (1995)

6. Aarnes, J.F., Rustad, A.B.: Probability and quasi-measures-a new interpretation. Math. Scand. 85(2), 278-284 (1999)

7. Andreou, T.: Density of Lipschitz functions. Proceedings of the conference of applied differential geometry-general relativity and the workshop on global analysis, differential geometry and Lie Algebras 2001, Balkan Society of Geometers, Geometry Balkan Press, 1-4 (2004)

8. Billingsley, P.: Convergence of Probability Measures, 2nd edn. John Wiley and Sons Inc, New York (1999)

9. Bogachev, V. I. : Measure Theory. vol. 2: Regular and Chaotic Dynamics, Izhevsk (2003). English transl.: Springer-Verlag, Berlin (2007)

10. Bogachev, V.I. : Weak convergence of measures. AMS Series: Mathematical surveys and monographs, vol. 234, Providence RI (2018)

11. Butler, S.V.: Density in the space of topological measures. Fund. Math. 174, 239-251 (2002)

12. Butler, S.V.: q-Functions and extreme topological measures. J. Math. Anal. Appl. 307, 465-479 (2005)

13. Butler, S. V.: Ways of obtaining topological measures on locally compact spaces. Bull. of Irkutsk State Univ., Series "Mathematics" (Izvestiya Irkutskogo Gosudarstvennogo Universiteta, Seriya "Matematika") 25, 33-45 (2018)

14. Butler, S.V.: Decompositions of signed deficient topological measures. Lett. Math. Sci. 1(1), 61-77 (2019)

15. Butler, S.V.: Non-linear functionals, deficient topological measures, and representation theorems on locally compact spaces. Banach J. Math. Anal. 14(3), 674-706 (2020)

16. Butler, S.V.: Integration with respect to deficient topological measures on locally compact spaces. Math. Slovaca 70(5), 1113-1134 (2020)

17. Butler, S. V.: Deficient topological measures on locally compact spaces. To appear in Math. Nachr. 294(6) (2021). https://doi.org/10.1002/mana.201800574

18. Butler, S. V.: Quasi-linear functionals on locally compact spaces. Accepted by Confluentes Mathematici. Preprint arXiv: 1902.03358

19. Butler, S. V.: Semisolid sets and topological measures. Preprint arXiv: 2103.09401

20. Denneberg, D.: Non-Additive Measure and Integral. Kluwer Academic Publishers, Dordrecht (1994)

21. Dickstein, A., Zapolsky, F.: Approximation of quasi-states on manifolds. J. Appl. Comput. Top. 3, 221-248 (2019)

22. Dugundji, J.: Topology. Allyn and Bacon Inc, Boston (1966)

23. Entov, M., Polterovich, L.: Quasi-states and symplectic intersections. Comment. Math. Helv. 81, 75-99 (2006)

24. Entov, M., Polterovich, L., Zapolsky, F.: Quasi-morphisms and the Poisson Bracket. Pure Appl. Math. Q. 4(3), 1037-1055 (2007)

25. Entov, M.: Quasi-morphisms and quasi-states in symplectic topology. Proceedings of the International Congress of Mathematicians, Seoul, 1147-1171 (2014)

26. Grubb, D.J., Laberge, T.: Spaces of quasi-measures. Canad. Math. Bull. 42(3), 291-297 (1999)

27. Johansen, $\varnothing$., Rustad, A.: Construction and properties of quasi-linear functionals. Trans. Amer. Math. Soc. 358(6), 2735-2758 (2006)

28. Kadison, R.V.: Transformation of states in operator theory and dynamics. Topology 3, 177-198 (1965)

29. Kechris, A.S.: Classical Descriptive Set Theory. Springer-Verlag, New York (1995)

30. Kelley, J.L.: General Topology. Springer-Verlag, Berlin (1955)

31. Knudsen, F.: Topology and the construction of extreme Quasi-measures. Adv. Math. 120(2), 302-321 (1996)

32. Mackey, G.W.: Quantum mechanics and Hilbert space. Amer. Math. Monthly 64, 45-57 (1957)

33. Mackey, G.W.: The Mathematical Foundations of Quantum Mechanics. Benjamin, New York (1963)

34. Parthasarathy, K.R.: Probability measures on metric spaces. Academic Press, New York (1967)

35. Pfanzagl, J.: Mathematical Statistics: Essays on History and Methodology. Springer, Berlin (2017)

36. Polterovich, L., Rosen, D.: Function theory on symplectic manifolds. CRM Monograph series, vol. 34. American Mathematical Society, Providence, RI (2014)

37. Rustad, A.B.: Unbounded quasi-integrals. Proc. Amer. Math. Soc. 129(1), 165-172 (2000)

38. Simon, B.: Real Analysis: A Comprehensive Course in Analysis. Part I. American Mathematical Society, USA (2015) 
39. Svistula, M.G.: A signed quasi-measure decomposition. Vestnik Samara Gos. Univ. Estestvennonauchn. 62(3), 192-207 (2008)

40. Svistula, M.G.: Deficient topological measures and functionals generated by them. Sbornik Math. 204(5), 726-761 (2013)

41. Svistula, M.G.: On integration with respect to a DT-measure. Positivity 20(3), 579-598 (2016)

42. von Neumann, J.: Mathematical Foundations of Quantum Mechanics. Princeton University Press, Princeton (1955). (Translation of Mathematische Grundlagen der Quantenmechanik. Springer, Berlin (1932).)

Publisher's Note Springer Nature remains neutral with regard to jurisdictional claims in published maps and institutional affiliations. 